\title{
A SEMANTIC APPROACH TO FAIRNESS
}

\author{
J.J.M.M. RUTTEN*
}

CWI, P.O. Box 4079, 1009 AB Amsterdam, The Netherlands

J.I. ZUCKER**

Department of Computer Science \& Systems McMaster University, Hamilton, Ont. L8S 4K1, Canada

\begin{abstract}
In the semantic framework of metric process theory, we undertake a general investigation of fairness of processes from two points of view: (1) intrinsic fairness of processes, and (2) fair operations on processes. Regarding (1), we shall define a "fairification" operation on processes called Fair such that for every (generally unfair) process $p$ the process Fair $(p)$ is fair, and contains precisely those paths of $p$ that are fair. Its definition uses systematic alternation of random choices. The second part of this paper treats the notion of fair operations on processes: suppose given an operator on processes (like merge, or infinite iteration), we want to define a fair version of it. For the operation of infinite iteration we define a fair version, again by a "fair scheduling" technique.

1980 Mathematical Subject classification: 68B10, 68C01, 68C05.

1986 Computing Reviews Categories: D.1.3, F.1.2

Key words and phrases: Fairness, semantic domains of metric processes, fair infinite iteration, alternation of random choices.
\end{abstract}

\section{INTRODUCTION}

The most basic context in which the notion of fairness can be defined is that of a repetitive choice among alternatives. In $[F]$ the reader can find an elaborate introduction to the notion(s) of fairness, with an extensive overview of the research in this

In this paper we propose a different approach, which could be called a semantic one, as opposed to the language (or syntax) directed approach mentioned above. Our

* The research of Jan Rutten was partially supported by ESPRIT project 415: Parallel Architectures and Languages for Advanced Information Processing - a VLSI-directed approach.

** The research of Jeffery Zucker was supported by the National Science Foundation under grant no. DCR-8504296 and by a grant from the Natural Sciences and Engineering Research Council of Canada. 
point of departure is a semantic domain for nondeterministic languages in general, without limiting ourselves to the choice of a particular language. Such a semantic domain will in general be a solution of some reflexive domain equation

$$
F P \cong P
$$

where $F$ is a functor on some category of mathematical domains, and " $\cong$ " means "is isomorphic to". Various techniques have been developed for solving this type of equation. We follow a metric approach, following Nivat $([\mathrm{N}])$ and De Bakker and Zucker ([BZ1]), and reformulated and extended in a category-theoretic setting in [AR]. The category $e$ under consideration consists of complete metric spaces, and the functors on $e$ are so-called contracting functors. These spaces are composed from basic metric spaces (sets provided with the trivial 0-1 metric) by the operations of union, Cartesian product, forming function spaces, and forming the set of all (closed) subsets of a given space. Examples would be complete metric spaces satisfying one of the following equations:

$$
\begin{aligned}
& P \cong A \cup(B \times P), \text { or } \\
& P \cong A \cup(B \rightarrow(C \times P)),
\end{aligned}
$$

where $A, B$ and $C$ are arbitrary sets and $\cong$ stands for "is isometric to". (Since elements of $e$ are pairs $\left\langle P, d_{P}\right\rangle$, consisting of a set $P$ and a metric $d_{P}$ on $P$, domain equations over $C$ should also specify a condition on these metrics. In this introduction, however, we omit such details.)

Another example of a domain is a metric space $P$ satisfying the domain equation:

$$
P \cong\left\{p_{0}\right\} \cup \Im_{c l}(B \times P) .
$$

(Here $\mathscr{P}_{c l}(\cdots)$ denotes the set of all closed subsets of $(\cdots)$.) Since this is the domain we shall use in this paper as a starting point for our study of fairness, we discuss it in some detail. The (possibly infinite) set $B=\left\{b_{1}, b_{2}, \ldots\right\}$ is called the alphabet of $P$. The elements of $P$ are called processes. A process $p \in P$ is either $p_{0}$, the so-called nil process, or a (closed) set of the form

$$
p=\left\{<b_{i}, p_{i}>\left|<b_{i}, p_{i}\right\rangle \in B \times P, i \in I\right\}
$$

for some set $I$ of indices. (Here the set $I$ represents the choice among alternatives.) Then $p$ can be regarded as a process that for each $i \in I$ can take a step $b_{i}$, and then continues with the process $p_{i}$ (called the resumption of $b_{i}$ ). This is itself either $p_{0}$, indicating that the process $p$ has terminated after performing step $b_{i}$, or again a (closed) set of possible next steps and corresponding resumptions.

Roughly, one can think of these processes as tree-like entities. However, there are some differences. Trees with a left branch labeled $a$ and a right branch labeled $b$, and 
with a left branch labeled $b$ and a right branch labeled $a$, are identified, and both are represented by $\left\{\left\langle a, p_{0}\right\rangle,\left\langle b, p_{0}\right\rangle\right\}$. A tree with only one branch labeled $a$ is identified with a tree with two branches both labeled $a$. Furthermore, we do not consider arbitrary subsets of $B \times P$, but only closed ones. For an extensive comparison of trees and processes we refer to [BK].

In our approach the elements of $B$, which are called basic steps, are atomic actions, whose possible interpretations have been abstracted from. One such interpretation would be to associate a basic step $b_{i}$ with each component of a guarded command, indicating that the $i$-th component of that command is selected. Another interpretation would be to regard $b_{i}$ as an arbitrary action of the $i$-th component of a system of (possibly infinitely many) active components, indicating that "progress" is being made by that component. A context in which this interpretation makes sense is that of object-oriented programming (see e.g. $[\mathrm{ABKR}]$ or $[\mathrm{C}]$ ). The basic steps could also be thought of as being different possible actions (e.g. read, write, assignment, etc.) which a single component can perform.

In this framework of metric process theory, we undertake a general investigation of fairness of processes from two points of view: (1) intrinsic fairness of processes, and (2) fair operations on processes.

Regarding (1), a process $p$ is called (intrinsically) fair if all its paths are fair. A path for $p$ is a sequence of pairs: $\left\langle a_{1}, p_{1}\right\rangle,\left\langle a_{2}, p_{2}\right\rangle, \ldots$, such that $\left\langle a_{1}, p_{1}\right\rangle \in p$ and $\left.<a_{i+1}, p_{i+1}\right\rangle \in p_{i}$ for all $i \geqslant 1$. The difference between fair and unfair paths can easily be illustrated with a simple example: consider a process $p \in P$ satisfying

$$
p=\{<0, p\rangle,<1, p\rangle\} .
$$

This process must choose infinitely often (in fact at every step) whether to perform the basic step " 0 " or the basic step " 1 ". The following path in $p$

$$
<0, p>,<0, p>,<0, p>, \ldots
$$

is unfair (with respect to basic step "l"), because step "l" never occurs whereas it can be taken infinitely often. An example of a fair path is

$$
<0, p>,\langle 1, p>,<0, p>,<1, p>, \ldots
$$

Actually, there are (at least) two notions of fairness current in the literature. The notion we are considering in this paper is often called "strong" fairness (e.g. in [OA]), as opposed to "weak" fairness. In our context a path $\pi$ would be called weakly fair if every basic step that is from some moment on continually enabled in $\pi$ occurs infinitely often in $\pi$. (For the definition of enabled see 2.3.) This notion is also called justice ([LPS]). A path is strongly fair if every basic step that is enabled infinitely often (but not necessarily continually) in $\pi$ occurs infinitely often in $\pi$.) The difference between these two notions can again be illustrated with a simple example: 
consider a process $p \in P$ satisfying

$$
p=\{<0,\{<0, p>\}>,<1,\{<1, p>\}>\} .
$$

This process can choose infinitely often whether to perform twice the basic step " 0 ", or twice the basic step "l". Then the path in $p$

$$
<0,\{<0, p>\}>,<0, p>,<0,\{<0, p>\}>,<0, p>, \ldots
$$

is weakly fair but not strongly fair. In this paper we only deal with strong fairness. The case of weak fairness can be handled similarly; in fact it would be even easier.

We shall define in section 3 (for a finite alphabet $B$ ) a "fairification" operation

$$
\text { Fair }: P \rightarrow P^{\text {Ind }}
$$

(where $P^{\text {Ind }}$ is a suitably extended version of $P$ ), such that the process Fair $(p)$ is fair, and contains precisely those paths of $p$ that are fair, or, more precisely, representatives of such paths. The relation between $\operatorname{Fair}(p)$ and $p$ will be clarified by the definition of a mapping from the paths of $\operatorname{Fair}(p)$ to those paths of $p$ which they represent. Roughly, $\boldsymbol{F a i r}(p)$ is defined by associating indices with the subprocesses (or "nodes") of $p$ so as to provide a "bookkeeping" of the way in which alternative subprocesses are chosen in forming paths. These indices indicate priorities for each of the basic steps $b_{j}$. During the construction of $\operatorname{Fair}(p)$, new sets of indices will from time to time be chosen by certain random choices. (This idea of implementing fair scheduling by means of systematic alternation of random choices is well known (see e.g. [AO], [BZ2,3], [P]).) In section 4 this theory is extended to an infinite alphabet, with an "expanding" system of indices (i.e. increasing in length), so that an index at a node records all the (finitely many) basic steps already encountered on the path to that node.

We turn now to (2), the notion of fair operations on processes. Suppose given an operation $\theta$ on processes, which is, say, binary: $\theta: P \times P \rightarrow P$. We want to define a fair version $\theta_{f}: P \times P \rightarrow P$ of $\theta$, such that for all $p_{1}, p_{2} \in P$ : first, if $p_{1}$ and $p_{2}$ are (intrinsically) fair, then so is $\theta_{f}\left(p_{1}, p_{2}\right)$; and second, $\Theta_{f}\left(p_{1}, p_{2}\right)$ is fair with respect to the operation $\theta$. This second condition must be explicated for each operation $\theta$. A good example is the merge operation $\|: P \times P \rightarrow P$. In [BZ2,3] a fair version $\|_{f}$ is defined. In this case the second condition is the requirement that all paths in the resulting process $p_{1} \|_{f} p_{2}$ must be fair with regard to alternate scheduling from $p_{1}$ and $p_{2}$. A trivial and wrong solution to the problem would be to define

$$
p_{1} \|_{f p_{2}}=\operatorname{Fair}\left(p_{1} \|_{p_{2}}\right) \text {. }
$$

Obviously, the first condition would be satisfied, but not so the second. The reason for this is, roughly, that in the resulting process $p_{1} \|_{f} p_{2}$, (intrinsically) unfair paths of $p_{1} \| p_{2}$ that are fair with respect to the alternate scheduling from $p_{1}$ and $p_{2}$ should 
still be present. The operation Fair, however, would remove them from $p_{1} l l p_{2}$. So this solution would be too coarse. A satisfactory solution was given in $[\mathrm{BZ} 2,3]$, where the fair merge was defined on the basis of alternate sequences of random choices.

In this paper (section 5) we shall consider another example of an operation on processes, namely infinite iteration $(\cdots)^{\omega}: P \rightarrow P$, defined by

$$
p^{\omega}=\lim _{n \rightarrow \infty} p^{n},
$$

where $p^{0}=p_{0}$ and $p^{n+1}=p^{n}$ op. (Here "o" stands for sequential composition of processes.) We define the fair infinite iteration $p^{\omega_{f}}$ of a process $p \in P$ and, after explicating the notion of fairness with respect to infinite iteration, prove that the conditions above are indeed satisfied. Our approach is flexible enough to enable us to deal here with two notions of fairness simultaneously: global and node fairness.

An area that remains to be investigated is that of fairness for non-uniform processes $[B Z 1]$, where our uninterpreted basic actions are replaced by basic state transformations, since here even the definition of fairness of paths in such processes is problematic.

Nevertheless an investigation of non-uniform processes would ultimately be necessary, to deal with certain issues which cannot be handled in the present framework. Consider, specifically, the case of guarded commands (with Boolean guards), where successive "visits" to the same node have different descendent nodes, depending on the state- for example, the process

$$
P::=*^{*}[b \rightarrow c:=\neg c \square b \wedge c \rightarrow b:=f f]
$$

which terminates only under (strong) fairness. Nodes corresponding to the "top level" here have, alternately, one and two descendents. In our "uniform" framework a node always has the same set of descendents, and so a situation like this cannot be handled.

RFLATED WORK: We already mentioned $[F]$ above, where the reader can find an introduction to the notion(s) of fairness. Next, we mention a few related papers without the intention of giving a complete overview of this area of research.

In [DM], fairness properties are imposed through metrics that allow convergence to fair processes only. The starting point is a simple concurrent language for which a semantics is given with the help of so-called concurrent histories, which are partial orderings describing 'true' concurrency. In [AO] and [CS], proof rules are given for fair transformations in concurrent systems: in the first paper for a fixed number of concurrent components, and in the latter for a (possibly) growing number.

The main difference between the above approaches and ours, is that we study fairness of processes purely at a semantic level. This enables us to consider the notion of arbitrary fair operation on processes, of which the merge (of concurrent, possibly infinitely many, processes) is just one example. 
ACKNOWLEDGEMENTS: It was Jaco de Bakker who first noticed that fair scheduling, implemented by systematic alternation of random choices (as in $[\mathrm{P}]$ ), could be used to model fair merge in the semantic framework of process domains, as in [BZ2,3]. The second author had useful discussions with Shenquan Xie on fairification and fair infinite iteration. We thank the anonymous referees for their detailed and constructive comments.

\section{MATHEMATICAL PRELIMINARIES}

\section{Definition 2.1 (Domains)}

We shall use mathematical domains $P$ of processes $p$, which are such that:

(1) $P$ is a complete metric space,

(2) $P$ satisfies the following reflexive equation:

$$
P \cong\left\{p_{0}\right\} \cup \mathscr{P}_{c l}(A \times P),
$$

where $\cong$ stands for "is isometric to", $p_{0}$ is a null process, $\mathscr{P}_{c l}(\cdots)$ denotes the set of all closed subsets of $(\cdots)$ and $A$, with typical elements $a$, is such that it contains as a subset a (possibly infinite) alphabet

$$
B=\left\{b_{1}, b_{2}, \ldots\right\}
$$

of basic steps.

We shall not dwell too long upon the mathematical details of the construction of a domain $P$ which satisfies the above definition. Let us just briefly mention two different approaches. First, one can take a metric completion of a union of metric spaces in the following way. We need two definitions. First, $d$ is an ultrametric on a metric space $M$ if, for all $x, y, z \in M$,

$$
d(x, z) \leqslant \max \{d(x, y), d(y, z)\}
$$

Second, given a metric space $(M, d)$, the metric $d$ induces a metric, the Hausdorff metric $d_{H}$, on the set $\mathscr{P}_{c l}(M)$ of closed subsets of $M$, defined by

$$
d_{H}(X, Y)=\max \left\{\sup _{\{x \in X\}}\left(\inf _{\{y \in Y\}} d(x, y)\right), \sup _{\{y \in Y\}}\left(\inf _{\{x \in X\}} d(x, y)\right)\right)
$$

Note that if $d$ is an ultrametric, then so is $d_{H}$.

Now we define a sequence of metric spaces $\left(\left(P_{n}, d_{n}\right)\right)_{n}$ by

$$
p_{0}=\left\{p_{0}\right\}, d_{0}\left(p_{0}, p_{0}\right)=0
$$




$$
\begin{aligned}
& P_{n+1}=p_{0} \cup \mathscr{P}_{c l}\left(A \times P_{n}\right) \\
& d_{n+1}\left(p_{0}, p_{0}\right)=0 \\
& d_{n+1}\left(p_{1} p_{0}\right)=d_{n+1}\left(p_{0}, p\right)=1, \text { for } p \neq p_{0}
\end{aligned}
$$

For $p, p^{\prime} \in P_{c l}\left(A \times P_{n}\right), d\left(p, p^{\prime}\right)$ is the Hausdorff metric induced by the distance between points $d_{n+1}(x, y)$, where, for $x=\left\langle a_{1}, p_{1}\right\rangle$ and $y=\left\langle a_{2}, p_{2}\right\rangle$,

$$
d_{n+1}(x, y)=\left[\begin{array}{lll}
1 & \text { if } a_{1} \neq a_{2} \\
1 / 2 \cdot d_{n}\left(p_{1}, p_{2}\right) & \text { if } a_{1}=a_{2}
\end{array}\right.
$$

Note that $P_{0} \subset P_{1} \subset \cdots$ and $d_{0} \subset d_{1} \subset \cdots$. Define

$$
P_{\omega}=\bigcup_{n} P_{n}, \quad d_{\omega}=\bigcup_{n} d_{n}
$$

and $(P, d)$ as the metric completion of $\left(P_{\omega}, d_{\omega}\right)$.

Note that $P$ is a complete metric space, $d$ is an ultrametric on $P$ with maximum value 1 , and $P$ satisfies the reflexive equation above. Full mathematical details and extensive motivation are supplied in [BZ1]. The second approach is to interpret the reflexive equation for $P$ as defining a functor $F$ on a category of complete metric spaces, thus:

$$
F P=\left\{p_{0}\right\} \cup \varphi_{c l}(A \times P) .
$$

(The definition of $F$ should also specify a metric for $F P$.) In $[\mathrm{AR}]$ it is shown how to define $F$ as a so-called contraction, which has a (unique) fixed point; so

$$
F P \sim P .
$$

Thus this method also presents us with a solution.

Rr:MARKs: We should be more precise about the metrics involved. We should have written the equation above as

$$
F P=\left\{p_{0}\right\} \cup \varphi_{C l}\left(A \times i d_{1 / 2}(P)\right),
$$

where, for any positive real number $c$, id $c$ maps a metric space $(M, d)$ onto $\left(M, d^{\prime}\right)$ with $d^{\prime}(x, y)=c \cdot d(x, y)$. For the details see [AR].

The metric $d$ on $P$ has the following convenient description: First, for $p \neq p_{0}$, we define the left projection of $p$ :

$$
\pi(p)=\left\{a \in A: \exists p^{\prime}\left[<a, p^{\prime}>\in p\right]\right\}
$$

Then we have, for $p, q \neq p_{0}$, the following two cases.

(1) if $\pi(p) \neq \pi(q)$ then $d(p, q)=1$ 


$$
\begin{aligned}
& \text { (2) if } \pi(p)=\pi(q) \\
& \text { then } d(p, q)=1 / 2 \sup \left\{d\left(p^{\prime}, q^{\prime}\right): \exists a \in A\left[<a, p^{\prime}>\in p \wedge<a, q^{\prime}>\in q\right]\right\}
\end{aligned}
$$

The constant $1 / 2$ appearing in the formula $\left({ }^{*}\right)$ will be used in the "contraction arguments" below.

We now introduce a number of concepts related to processes.

Definition 2.2 (Paths)

A path for a process $p \in P$ is a (finite or infinite) sequence

$$
\left.\left.\pi=\left(<a_{1}, p_{1}\right\rangle,<a_{2}, p_{2}\right\rangle, \ldots\right)
$$

such that

$$
<a_{1}, p_{1}>\in p \wedge \forall i \geqslant 1\left[<a_{i+1}, p_{i+1}>\in p_{i}\right] .
$$

We say that $\pi$ passes through $p_{i}$, and $p_{i}$ will be called a node of $p$ or a subprocess of $p$ (for $i \geqslant 1$ ). The set of all paths for $p$ will be called Paths $(p)$.

The following definition explains which processes we want to consider fair.

\section{Definttion 2.3 (Fairness)}

(a) Let $b_{i} \in B$. Consider a path

$$
\pi \equiv\left(<a_{1}, p_{1}>,<a_{2}, p_{2}>, \ldots\right) .
$$

We say that $b_{i}$ is enabled in $\pi$ (or $i$ is enabled in $\pi$ ) at step $k$ whenever

$$
\left.\exists k \in \mathbb{N} \exists q \in P\left[<b_{i}, q\right\rangle \in p_{k}\right] .
$$

We say that $b_{i}$ occurs in $\pi$, whenever

$$
\exists k \in \mathbb{N}\left[a_{k}=b_{i}\right] .
$$

(b) We call a path $\pi$ fair whenever for all $b_{i} \in B$, if $b_{i}$ is enabled infinitely often in $\pi$, then it occurs infinitely often in $\pi$.

(c) A process $p \in P$ is called fair if all its paths are fair.

EXAMPLE: Let $p \in P$ be such that $p=\{\langle a, p\rangle,\langle b, p\rangle\}$. Then $b$ is continually enabled in

$$
\pi=(<a, p>,<a, p>, \ldots),
$$

but never occurs in it. Thus, the path $\pi$ is unfair. 
Please note that only basic steps $b_{i} \in B$ are taken into account in the definition of fairness.

\section{FAIRIFICATION OF PRocesses WITH FINITE ALPHABET}

Let $P$ be defined by

$$
P \cong\left\{p_{0}\right\} \cup \mathscr{P}_{c l}(B \times P)
$$

with $B$ a finite alphabet:

$$
B=\left\{b_{1}, \ldots, b_{m}\right\} .
$$

Given a process $p \in P$, we want to form a new process $\operatorname{Fair}(p)$, which is, in some sense, a fair version of $p$. For this purpose we want to define a function

$$
\text { Fair: } P \rightarrow P^{\text {Ind }}
$$

such that there is an obvious correspondence between the paths of $\operatorname{Fair}(p)$ and the fair paths of $p$. Here $P^{\text {Ind }}$ is given by:

$$
P^{\text {Ind }}=\left\{p_{0}\right\} \cup \rho_{c l}\left(A \times P^{\text {Ind }}\right),
$$

where $A=B \cup$ Index, and Index is a set of indices (to be defined below). A node $p^{\prime}$ of a process $p \in P^{I n d}$ with

$$
p^{\prime}=\left\{<\nu, p_{\nu}>\mid \nu \in I\right\},
$$

for some subset $I$ of Index, is called a sum node and is denoted by

$$
p^{\prime}=\sum_{\nu \in I} p_{\nu}
$$

After having defined the function Fair, we shall clarify the relation between $p$ and Fair $(p)$ by defining a mapping

$$
\Phi: \text { Paths }(\text { Fair }(p)) \rightarrow \text { Paths }(p),
$$

that will satisfy the following two properties. First, for every path $\pi \in \boldsymbol{P a t h s}(\boldsymbol{F a i r}(p))$ we have that $\Phi(\pi)$ is fair. Secondly, any fair path in $p$ will be in the range of $\Phi$. The function Fair will be defined in such a way that it transforms a process $p$ into a fair process Fair (p) by labeling each node of $p$ with an index and, moreover, interspersing some new nodes consisting of sums of indices (to be defined below). Indices are the main building blocks in the definition of the function Fair. They are defined as 
follows.

Definition 3.1 (Indices)

The set Index of indices, with typical elements $\nu$, is given by

$$
\begin{aligned}
\text { Index }= & \left\{<n_{1}^{s_{1}}, \ldots, n_{m}^{s_{m}}>1\right. \\
& \left.\forall i \in\{1, \ldots, m\}\left[n_{i} \geqslant 0 \wedge 0<s_{i} \leqslant \infty \wedge\left(n_{i}=0 \Leftrightarrow s_{i}=\infty\right)\right]\right\},
\end{aligned}
$$

where $m$ is the number of elements in $B$, and $n_{i}^{s_{i}}$ denotes the Cartesian pair $<n_{i}, s_{i}>$.

Let $p$ be a process and $\nu$ an index. The process $p^{\nu}$, which is defined below, can be viewed, informally speaking, as a process that behaves like $p$ as far as is allowed by the index $\nu$. Consider the $i$-th element of $\nu$, say $n_{i}^{s_{i}}$. It is related to $b_{i}$, the $i$-th element of our alphabet $B$. The interpretation of $n_{i}^{s_{i}}$ (relative to $p$ ) is that in paths starting in $p$, a step $b_{i}$ is permitted $n_{i}$ times with priority $s_{i}$.

For the priorities $s_{i}$ we have the convention that a low number indicates a high priority. It is possible that two or more $s_{i}$ 's have the same value, the corresponding $b_{i}$ 's having the same priority. The symbol $\infty$ indicates the lowest priority possible. Because it is always associated with an $n$ that is 0 , it can also be interpreted as indicating no priority at all.

\section{REMARK}

The interpretation of the $i$-th component $n_{i}^{S_{i}}$ is in a sense orthogonal to the approach taken in e.g. [AO]. There a single number $z_{i}$ is used to indicate the priority of the $i$-th component of some system of active components. This number $z_{i}$ indicates, roughly, the number of times a computation can "allow itself" not to choose this component as the next one to make progress. In our approach the number $n_{i}$ indicates the number of times we are allowed to choose $b_{i}$ (the $i$-th component) as the next step, before another component gets the highest priority.

Now suppose we have a process $p$ containing a step $\left\langle b_{i}, q\right\rangle$ :

$$
p=\left\{\ldots,<b_{i}, q>, \ldots\right\} ;
$$

and assume furthermore that we have $\nu \in$ Index with

$$
\nu=\left\langle\ldots, n_{i}^{s_{i}}, \ldots\right\rangle
$$

where $n_{i}>0$ and $s_{i}=\min \left\{s_{1}, \ldots, s_{m}\right\}$. Then, according to our interpretation of $p^{\nu}$, it is permitted to choose $\left\langle b_{i}, q\right\rangle$ as the first step of a path starting from $p$. With the resumption $q$ of this step will be associated a new index $\nu^{-}[i]$, in which $n_{i}$ is decreased by one. If $n_{i}>1$ nothing happens to the priority $s_{i}$ of $b_{i}$. If $n_{i}=1$ (and so 
decreased to 0 ) it is, for the time being, the last time that $b_{i}$ is allowed, and $s_{i}$ is changed to $\infty$ (the lowest priority possible). As we will see, at some later stage it will be taken care of that $n_{i}$ and $s_{i}$ are reset again, so that $n_{i}>0$ and $s_{i}<\infty$. All this is formalized in the following definition.

\section{DefinITION 3.2}

Let $\nu \in$ Index be such that

$$
\nu=\left\langle n_{1}^{s_{1}}, \ldots, n_{i}^{s_{i}}, \ldots, n_{m}^{s_{m}}\right\rangle
$$

and let $i \in\{1, \ldots, m\}$. We define

$$
\nu^{-}[i]=\left[\begin{array}{ll}
\left.<n_{1}^{s_{1}}, \ldots,\left(n_{i}-1\right)^{s_{i}}, \ldots, n_{m}^{s_{m}}\right\rangle & \text { if } n_{i}>1 \\
\left\langle n_{1}^{s_{1}}, \ldots, 0^{\infty}, \ldots, n_{m}^{s_{m}}\right\rangle & \text { if } n_{i}=1 \\
\text { undefined } & \text { if } n_{i}=0 .
\end{array}\right.
$$

There is another operation on indices we shall need.

\section{Definition 3.3}

Let $\nu \in$ Index be such that

$$
\nu=\left\langle n_{1}^{s_{1}}, \ldots, n_{m}^{s_{m}}\right\rangle
$$

then

$$
\begin{aligned}
N(\nu)= & \left\{<\tilde{n}_{1}^{\tilde{s}_{1}}, \ldots, \tilde{n}_{m} \tilde{s}_{m}>1\right. \\
& \forall j \in\{1, \ldots, m\} \\
& {\left[\left(n_{j}=0 \wedge s_{j}=\infty\right) \Rightarrow\left(\tilde{n}_{j}>0 \wedge \tilde{s}_{j}=\right) \max \left(\left\{s_{1}, \ldots, s_{m}\right\} \backslash\{\infty\}\right)+1\right) \wedge } \\
& \left.\left.\left(n_{j}>0 \wedge s_{j}<\infty\right) \Rightarrow\left(\tilde{n}_{j}=n_{j} \wedge \tilde{s}_{j}=s_{j}\right)\right]\right\}
\end{aligned}
$$

The elements $\tilde{\nu}$ in $N(\nu)$ are obtained from $\nu$ by changing, for all $i$ with $n_{i}=0$ and $s_{i}=\infty$, the value of $n_{i}$ to an arbitrary positive number and the value of $s_{i}$ to $s+1$. In words, this means that $b_{i}$ is again allowed to be chosen ( $\tilde{n}_{i}$ times) but with a priority lower than all other priorities present in $\nu$ that are not $\infty$. This definition will also be used in the definition of Fair, where it will be further elucidated.

We now give this definition, upon which an explanation will follow.

\section{Definirion 3.4 (Fairification)}

We define a function 
Fair $P \rightarrow P^{\text {Ind }}$.

Let $p \in P$. Then

$$
\operatorname{Fair}(p)=\sum_{\nu \in I_{0}} \text { fair }(p, \nu),
$$

where

$$
I_{0}=\left\{\left\langle n_{1}^{1}, \ldots, n_{m}^{1}\right\rangle \mid n_{i}>0, i=1, \ldots, m\right\}
$$

and

$$
\text { fair } P \times \text { Index } \rightarrow P^{\text {Ind }}
$$

is defined as follows. (We often write $p^{\nu}$ for $f a i r(p, \nu)$.) For all $\nu \in$ Index we define

$$
\operatorname{fair}\left(p_{0}, \nu\right)=p_{0}
$$

For $p \neq p_{0}$ we distinguish two cases.

Case 1:

$$
\begin{aligned}
& \text { If } \exists i \in\{1, \ldots, m\}\left[n_{i}>0 \wedge s_{i}<\infty \wedge \text { enabled }(i)\right] \\
& \text { then } p^{\nu}=\left\{<b_{j}, q^{\nu^{-}[j]}>\mid<b_{j}, q>\in p \wedge s_{j}=\min \left\{s_{1}, \ldots, s_{m}\right\}\right\} \text {. }
\end{aligned}
$$

Case 2:

$$
\begin{aligned}
& \text { If } \forall i \in\{1, \ldots, m\}\left[\operatorname{enabled}(i) \Rightarrow\left(n_{i}=0 \wedge s_{i}=\infty\right)\right] \\
& \text { then } p^{\nu}=\sum_{\tilde{\nu} \in N(\nu)} p^{\tilde{\nu}}
\end{aligned}
$$

\section{REMARKS}

(1) The definition of fair: $P \times$ Index $\rightarrow P^{\text {Ind }}$ is self-referential and therefore needs some justification. We observe that fair could be defined as the fixed point of a mapping

$$
\Phi:\left(P \times \text { Index } \rightarrow P^{\text {Ind }}\right) \rightarrow\left(P \times \text { Index } \rightarrow P^{\text {Ind }}\right),
$$

which can be defined according to the definition scheme of fair above. It is straightforward to show that such a definition yields a contracting function (as we will see), which thus has a unique fixed point (cf. Banach's fixed point theorem ([E], [BZ1])). We now show that $\Phi$ is a contracting function using equation $\left(^{*}\right)$ in Remark 2 in Section 2. First note that the distance between two functions

$$
\phi, \psi: P \times \text { Index } \rightarrow P^{\text {Ind }}
$$


is given by

$$
d(\phi, \psi)=\sup \{d(\phi(p, \nu), \psi(p, \nu)): p \in P, \nu \in \operatorname{Index}\}
$$

So

$$
d(\Phi(\phi), \Phi(\psi))=\sup \{d(\Phi(\phi)(p, \nu), \Phi(\psi)(p, \nu)): p \in P, \nu \in \operatorname{Index}\}
$$

Now for all $p \in P, \nu \in$ Index

$$
\begin{aligned}
& d(\Phi(\phi)(p, \nu), \Phi(\psi)(p, \nu))=\quad \text { (following Definition } 3.4 \text { schematically) } \\
& \text { IF } p=p_{0} \text { THEN } d\left(p_{0}, p_{0}\right)=0 \\
& \text { ELSE IF [Case 1] THEN } 1 / 2 \sup \left\{d\left(\phi\left(q, \nu^{-}[j]\right), \psi\left(q, \nu^{-}[j]\right)\right): \cdots\right\} \text { by }\left(^{*}\right) \\
& \leqslant 1 / 2 d(\phi, \psi) \text { by }(1) \\
& \leqslant 1 / 2 d(\phi, \psi) \text { by }(1)
\end{aligned}
$$

Hence

$$
d(\Phi(\phi), \Phi(\psi)) \leqslant 1 / 2 d(\phi, \psi) \text { by }(2)
$$

(2) Because case 2 never occurs twice in succession, fair $(p, \nu)$ never contains two sum nodes successively.

(3) Every node in $\operatorname{Fair}(p)$ is either a sum node, or of the form $\left\{\left\langle b_{i_{j}}, p_{j}\right\rangle \mid j \in I\right\}$, for some set of indices $I$.

(4) We give some informal intuition for this definition. The indices $\nu \in$ Index in the definition above can be interpreted as strategies for the construction of a process Fair $(p)$ such that every path in this process will be fair with respect to every $b_{i}$ in $B$. An element $\nu$ in $I_{0}$ can be regarded as permission, for each $i$, to choose $b_{i}$ $n_{i}$ times. All $i$ are supplied at the beginning with the same priority, that is 1 .

We will treat $p^{\nu}$ for the case that $p \neq p_{0}$. As long as case 1 applies there is no need to change our strategy or, in other words, to choose a new $\nu$. Each $b_{i}$ that is enabled at $p$, and for which $n_{i}>0$ and $s_{i}=\min \left\{s_{1}, \ldots, s_{m}\right\}$, may be chosen as the next step in the new process we are constructing. The index $\nu$ is changed according to the definition of $\nu^{-}[i]$, so $n_{i}$ is decreased by 1 and the priority $s_{i}$ remains constant, unless $n_{i}$ was 1 . Then it is set to $\infty$, indicating no priority at all. Because every application of case 1 causes the decrease of an $n_{i}$, it is obvious that after a finite number of such applications case 2 must hold. For didactic purposes we shall now make a conceptual distinction between two possible situations that may arise in this case. Formally however, as may be inferred from the definition of case 2 , this is not necessary. 
First, it may be the case that all $n_{i}$ 's have been decreased to 0 (and all $s_{i}$ 's have been set to $\infty$ ). Then we can consider the strategy suggested by the $\nu$ we started with to be a great success: every $b_{i}$ has been chosen the number of times we had in mind for it $\left(n_{i}\right)$. The fact that originally all $n_{i}$ 's were strictly positive implies that so far we have made sure that all $b_{i}$ 's have been treated fairly. It is clear what to do next: we can just restart by choosing a new index $\nu$, with all $n_{i}$ strictly positive and all $s_{i}$ set to 1 . According to the definition of $N(\nu)$, this is exactly what happens in this case.

The second situation is more typical. It concerns the case that for all $i$ that are enabled at $p, n_{i}=0$ and $s_{i}=\infty$. But we have not finished the strategy suggested by the original $\nu$, because there exists at least one $j$ not enabled at $p$, with $n_{j}>0$ and $s_{j}<\infty$. Although we have not finished our first strategy, we are forced to change it because it does not tell us what to do about the $i$ 's that are enabled at p. A new strategy $\tilde{\nu}$ is defined such that for all $j$ with $n_{j}>0$ and $s_{j}<\infty$ these values remain unchanged, thus preserving that part of the first strategy $(\nu)$ that has not yet been dealt with. For all other $i$ (enabled or not enabled) the value of $n_{i}$ is set to an arbitrary strictly positive number, and the value of $s_{i}$ to $\max \left\{s_{1}, \ldots, s_{m}\right\}+1$. So the new priority introduced here is lower than all the already existing priorities. When at a later stage one of the $j$ s, for which $n_{j}$ and $s_{j}$ remain unchanged here, is enabled, it will take precedence over those $i$ 's for which a new priority is introduced. Thus a fair treatment of such $j$ s is ensured for the future.

Now for the rest of this section let $p \in P$ be fixed. We define a mapping

$$
\Phi: \text { Paths }(\text { Fair }(p)) \rightarrow \text { Paths }(p),
$$

relating to each path $\pi$ in $\operatorname{Fair}(p)$ a fair path in $p$. For its formal definition we shall make use of the following lemma.

LEMMA 3.5

For all $\bar{p} \in P$ with $\bar{p} \neq p_{0}, \nu \in$ Index and $\langle a, q\rangle \in$ fair $(\bar{p}, \nu)$, there exist $p^{\prime} \in P$ and $\nu^{\prime} \in$ Index such that

$$
\begin{aligned}
& q=\operatorname{fair}\left(p^{\prime}, \nu^{\prime}\right) \wedge \\
& a \in \text { Index } \Rightarrow p^{\prime}=\bar{p} \wedge \\
& a \in B \Rightarrow<a, p^{\prime}>\in \bar{p} .
\end{aligned}
$$

The proof is straightforward from the definition of $\bar{p}^{\nu}(=$ fair $(p, \nu))$.

DEFINITION 3.6 (The mapping $\Phi$ ) 
Let

$$
\pi=\left\langle a_{0}, q_{0}\right\rangle,\left\langle a_{1}, q_{1}\right\rangle, \ldots
$$

be a path in Fair(p). By the above lemma and the definition of Fair(p) we can rewrite it as

$$
\pi=\left\langle a_{0}, p^{\nu}\right\rangle,\left\langle a_{1}, p_{1}^{\nu_{1}}\right\rangle, \ldots,
$$

for certain $\nu, \nu_{1}, \ldots \in$ Index and $p_{1}, p_{2}, \ldots \in P$. Now if we delete all pairs $\left\langle a_{i}, p_{i}^{\nu_{i}}\right\rangle$ with $a_{i} \in$ Index, and all superscripts $\nu_{i}$, we get a sequence

$$
\left.\Phi(\pi)=<a_{i_{1}}, p_{i_{1}}\right\rangle,<a_{i_{2}}, p_{i_{2}}>, \ldots,
$$

which is a path in $p$. We call $\Phi(\pi)$ the path in $p$ corresponding to the path $\pi$ in Fair $(p)$. This defines a mapping

$$
\Phi: \text { Paths }(\text { Fair }(p)) \rightarrow \text { Paths }(p) .
$$

\section{EXAMPLE}

Consider the alphabet $B=\{0,1\}$ and the process $p \in P$ defined by

$$
p \cong\{<0, p>,<1, p>\}
$$

We give an example of a path $\pi$ in $\operatorname{Fair}(p)$ :

$$
\begin{aligned}
& \pi= \\
& <3^{1}, 2^{1}>, \text { fair }\left(<3^{1}, 2^{1}>, p\right)>, \\
& <1, \text { fair }\left(<3^{1}, 1^{1}>, p\right)> \\
& <0, \text { fair }\left(<2^{1}, 1^{1}>, p\right)>, \\
& <1, \text { fair }\left(<2^{1}, 0^{\infty}>, p\right)> \\
& <0, \text { fair }\left(<1^{1}, 0^{\infty}>, p\right)> \\
& <0, \text { fair }\left(<0^{\infty}, 0^{\infty}>, p\right)>, \\
& <<23^{1}, 183^{1}>, \text { fair }\left(<23^{1}, 183^{1}>, p\right)>, \cdots
\end{aligned}
$$

For this $\pi$ we have

$$
\Phi(\pi)=\langle 1, p>,<0, p>,<1, p>,<0, p>,<0, p>, \cdots
$$

Next, we have an important theorem. 
THEOREM 3.7

Fair $(p)$ is fair. That is, for all $\pi \in \operatorname{Paths}(\boldsymbol{F a i r}(p)), \pi$ is fair.

ProOF

Let $\pi \in$ Paths $($ Fair $(p))$ be such, that

$$
\begin{aligned}
\pi & =\left\langle a_{1}, q_{1}\right\rangle,\left\langle a_{2}, q_{2}\right\rangle, \ldots \\
& =\left\langle a_{1}, p_{1}^{\nu_{1}}\right\rangle,\left\langle a_{2}, p_{2}^{\nu_{2}}\right\rangle, \ldots
\end{aligned}
$$

Suppose $b_{i}$ is enabled infinitely often in $\pi$. We must show that $b_{i}$ occurs infinitely often within $\pi$. It is sufficient to show that for any $j$, if $b_{i}$ is enabled at the node $p_{j}^{p_{j}}$ of $\pi$, then $b_{i}$ occurs further on in the path $\pi$, that is, for some $j^{\prime} \geqslant j: b_{i}=a_{j^{\prime}}$.

We consider the sequence $\nu_{j}, \nu_{j+1}, \ldots$ and observe that for every $k \in \mathbb{N}, \nu_{k+1}$ is obtained from $\nu_{k}$ by an application of case 1 or 2 in the definition of fair $(p, \nu)$ (definition 3.4). Now let

$$
\nu_{j}=\left\langle n_{1}^{s_{1}}, \ldots, n_{m}^{s_{m}}\right\rangle .
$$

We consider all possible cases.

(1) $n_{i}=0$ :

Then $s_{i}=\infty$. For every application of case 1 (above) onc of the $n_{k}$ 's must decrease. Therefore eventually case 2 must apply, which makes all $n_{k}$ 's positive and brings us to the next case.

(2) $n_{i}>0$ : This implies $s_{i}<\infty$. As long as $s_{i}$ is not the highest priority, the following may happen. Any application of case 1 results in either the decrease of an $n_{k}$, not to 0 , or the decrease of an $n_{k}$ to 0 and the removal of a higher priority than $s_{i}$. After a finite number of applications of case 1, the latter must happen. Any application of case 2 introduces only priorities that are lower than $s_{t}$, and must be followed by an application of case 1. Furthermore, during any of these applications, $n_{i}$ and $s_{i}$ remain constant. It follows then that eventually $s_{i}$ will be the highest priority. Because $b_{i}$ is enabled infinitely often in $\pi$, it must be enabled at some step beyond this, at which point case 1 will be applied to it and $b_{t}$ will occur at the next step.

Now that we have proved that we did not promise too much, that is to say that Fair $(p)$ indeed contains only fair paths, let us also make sure that for all fair paths in $p$ there is a corresponding path in $\operatorname{Fair}(p)$.

Theorem 3.8

Any fair path in $p$ is in the range of the mapping $\Phi$. 
Proof

Given a fair path $\pi^{\prime} \in \operatorname{Paths}(p)$, we must construct a path $\pi \in \operatorname{Paths}(\boldsymbol{F a i r}(p))$ such that

$$
\Phi(\pi)=\pi^{\prime} .
$$

First, we partition the set $\{1, \ldots, m\}$ into two parts $F$ and $I$, where $F$ is the set of all $i$ such that $b_{i}$ is enabled finitely often (perhaps never) in $\pi^{\prime}$, and $I$ is the set of all $i$ such that $b_{i}$ is enabled infinitely often in $\pi^{\prime}$. Thus:

$$
\{1, \ldots, m\}=I \cup F \text {. }
$$

Note that for all $i \in F, b_{i}$ occurs only finitely often in $\pi^{\prime}$, and for all $i \in I, b_{i}$ occurs infinitely often in $\pi^{\prime}$, since $\pi^{\prime}$ is fair. Let $l_{1} \in \mathbb{N}$ be so big that

(1) no $b_{i}$ with $i \in F$ is enabled in the part of $\pi^{\prime}$ at or after step $l_{1}$;

(2) every $b_{i}$ with $i \in I$ occurs at least once by then.

Now for $i=1, \ldots, m$, let $n_{i}{ }^{\prime}$ be the number of times that $b_{i}$ occurs before (or at) step $l_{1}$ and then define

$$
n_{i}=\left[\begin{array}{ll}
n_{i}{ }^{\prime}+1 & \text { if } i \in F \\
n_{i}{ }^{\prime} & \text { if } \quad i \in I .
\end{array}\right.
$$

We define our first index $\nu_{1}$ by

$$
\nu_{1}=\left\langle n_{1}^{1}, \ldots, n_{m}^{1}\right\rangle \text {. }
$$

Now we can construct the first part of the path $\pi$ corresponding with the part of $\pi^{\prime}$ before step $l_{1}$, by starting with $p^{\nu}$, and repeatedly applying case 1 for the appropriate $b_{i}$, thus decreasing the $n_{i}$ 's until (at step $l_{1}$ ) our index is such that for all $i \in\{1, \ldots, m\}$ :

$$
\begin{aligned}
& i \in F \Rightarrow n_{i}=1 \wedge s_{i}=1, \\
& i \in I \Rightarrow n_{i}=0 \wedge s_{i}=\infty .
\end{aligned}
$$

Now case 2 must be applied to get a sum node, since no $i \in F$ is enabled at step $l_{1}$. To determine the following index $\nu_{2}$ we again choose a number $l_{2} \in \mathbb{N}$, with $l_{2}>l_{1}$, such that every $b_{i}$ with $i \in I$ occurs at least once between steps $l_{1}$ and $l_{2}$ (including $l_{1}$, excluding $l_{2}$ ). Then choose an index $\nu_{2}$ such that, for $i \in I, n_{i}$ denotes the number of occurrences of $b_{i}$ between $l_{1}$ and $l_{2}$. We proceed as before, constructing the part of $\pi^{\prime}$ between $l_{1}$ and $l_{2}$. Continuing in this way, we construct a path $\pi$ in $\operatorname{Fair}(p)$ such that $\Phi(\pi)=\pi^{\prime}$.

REMARK: This function $\Phi$ is not bijective. In general there are more than one (in fact, infinitely many) paths in $\operatorname{Fair}(p)$ that are mapped by $\Phi$ to the same path in $p$. 


\section{FAIRIFICATION OF PROCESSES WITH INFINITE ALPHABET}

We now want to extend our technique of fairification to a set of processes, which we shall (again) call $P$, defined by

$$
P \cong\left\{p_{0}\right\} \cup \mathscr{P}_{c l}(B \times P)
$$

with $B$ an infinite denumerable alphabet:

$$
B=\left\{b_{1}, b_{2}, \ldots\right\} \text {. }
$$

We shall again define a function

$$
\text { Fair }: P \rightarrow P^{\text {Ind }} \text {, }
$$

where $P^{I n d}$ is given by

$$
\begin{aligned}
& P^{\text {Ind }}=\left\{p_{0}\right\} \cup \Theta_{c l}\left(A \times P^{I n d}\right), \\
& A=B \cup \text { Index, }
\end{aligned}
$$

with Index to be defined below. We shall repeat the approach of the previous section with some small but essential changes. The definitions, lemmas and theorems that need not be changed will be mentioned, but not repeated in full.

An important change is the new definition of indices. They no longer have a fixed length.

\section{Definition 4.1 (Indices)}

The set Index of indices, with typical elements $\nu$, is given by

$$
\text { Index }=\cup_{m \in \mathbb{N}} \text { Index }^{[m]},
$$

with

$$
\begin{aligned}
& \text { Index }^{[m]}= \\
& \left\{<n_{1}^{s_{1}}, \ldots, n_{m}^{s_{m}}>\mid \forall i \in\{1, \ldots, m\}\left[n_{i} \geqslant 0 \wedge 0<s_{i} \leqslant \infty \wedge\left(n_{i}=0 \Leftrightarrow s_{i}=\infty\right)\right]\right\} .
\end{aligned}
$$

An index of length $k$ is related to the first $k$ elements of our alphabet $B$. The interpretation of $n_{i}$ and priority $s_{i}$ is as before. When we define, for a given process $p$, a fair version $\operatorname{Fair}(p)$, we shall, during the construction, increase the length of the indices used, thus considering fairness with respect to a growing number of basic steps $b_{i}$. Once the length of an index is bigger than or equal to some $i \in \mathbb{N}$, it is ensured that $b_{i}$ is treated fairly thereafter. The definition of the first operation on indices, $\nu^{-}[\cdots]$, remains unchanged, but for the fact that the original definition 
(3.2) should hold for indices of arbitrary length. The most important adaptation of this section lies in the following new definition of $N(\nu)$.

\section{Definition 4.2}

Let $\nu \in$ Index be such that $\nu=\left\langle n_{1}^{s_{1}}, \ldots, n_{m}^{s_{m}}\right\rangle$ and let $p \in P$. We define

$$
\begin{aligned}
N(\nu, p)=\{ & <\tilde{n}_{1}^{\tilde{s}_{1}}, \ldots, \tilde{n}_{m^{\prime}}>1 \\
& m^{\prime}>m \wedge \\
& \left\{k \mid 1 \leqslant k \leqslant m^{\prime} \wedge \tilde{n}_{k}>0 \wedge k \text { enabled at } p\right\} \neq \varnothing \wedge \\
& \forall j\left[\left((1 \leqslant j \leqslant m) \wedge n_{j}=0 \wedge s_{j}=\infty\right) \Rightarrow\right. \\
& \left.\left(\tilde{n}_{j}>0 \wedge \tilde{s}_{j}=\max \left\{s_{1}, \ldots, s_{m}\right\}+1\right)\right) \wedge \\
& \left.\left((1 \leqslant j \leqslant m) \wedge n_{j}>0 \wedge s_{j}<\infty\right) \Rightarrow\left(\tilde{n}_{j}=n_{j} \wedge \tilde{s}_{j}=s_{j}\right)\right) \wedge \\
& \left.\left.\left.m<j \leqslant m^{\prime} \Rightarrow\left(\tilde{n}_{j}>0 \wedge \tilde{s}_{j}=s+1\right) \vee\left(\tilde{n}_{j}=0 \wedge \tilde{s}_{j}=\infty\right)\right)\right]\right\}
\end{aligned}
$$

Let us see how this definition is used in the definition of the function Fair below, and then try to comment on its intuitive interpretation. Although we do not change the definition of Fair (definition 3.4), we repeat its most interesting part and discuss it in the context of the altered definition of $N(v)$.

If $p \in P$ with $p \neq p_{0}$, then $p^{\nu}(=f a i r(p, \nu))$ is given by:

Case 1:

$$
\begin{aligned}
& \text { If } \exists i \in\{1, \ldots, \text { length }(\nu)\}\left[n_{i}>0 \wedge s_{i}<\infty \wedge \text { enabled }(i)\right] \\
& \text { then } p^{\nu}=\left\{<b_{j}, q^{\nu^{-}[j]}>\mid<b_{j}, q>\in p \wedge s_{j}=\min \left\{s_{1}, \ldots, s_{\text {length }(\nu)}\right\}\right\} .
\end{aligned}
$$

Case 2:

$$
\begin{aligned}
& \text { If } \forall i \in\{1, \ldots, \text { length }(\nu)\}\left[\operatorname{enabled}(i) \Rightarrow\left(n_{i}=0 \wedge s_{i}=\infty\right)\right] \text {, } \\
& \text { then } p^{\nu}=\sum_{\tilde{\nu} \in N(\nu, p)} p^{\tilde{\nu}} .
\end{aligned}
$$

The interpretation of case 1 is the same as before. When the condition of case 2 holds, we are obliged to change our strategy, that is to choose a new index, because our current strategy does not say anything about the $i$ 's that are enabled at $p$. This can have two reasons. For such an $i$ we either have $n_{i}=0$ and $s_{i}=\infty$ or $i>$ length $(\nu)$. 
In order to be able to continue our construction, we therefore allow several new strategies $\tilde{\nu} \in N(\nu, p)$, which all must satisfy the following constraints. First, the part of the old strategy $\nu$ that has not been dealt with yet has to be preserved: for $1 \leqslant i \leqslant$ length $(\nu)$ with $n_{i}>0$ and $s_{i}<\infty$ we have $\tilde{n}_{i}=n_{i}$ and $\tilde{s}_{i}=s_{i}$. Then, for $1 \leqslant i \leqslant$ length $(\nu)$ with $n_{i}=0$ and $s_{i}=\infty$, the values of $n_{i}$ and $s_{i}$ are reset: $\tilde{n}_{i}$ arbitrary positive, $\tilde{s}_{i}=1+s$. As in the finite case, the new priority is lower than the existing ones. Because we want each $b_{k} \in B$ eventually to be treated fairly, for each $k$ there should be a moment in our construction where an index $\nu$ is introduced with length $(\nu)>k$. Therefore we require the length of the new index $i$ to be strictly greater than the length of $\nu$. For the newly introduced $j^{\prime} s($ length $(\nu)<j \leqslant m$ ) we require

$$
\left(\tilde{n}_{j}>0 \wedge \tilde{s}_{j}=s+1\right) \vee\left(\tilde{n}_{j}=0 \wedge \tilde{s}_{j}=\infty\right) .
$$

Although here $\tilde{n}_{j}=0$ is allowed, we know that the next time that case 2 is applied $\tilde{n}_{j}$ will be set to a strictly positive value. The newcomers, so to speak, are granted one (and only one) moment of respite. The motivation for this generosity lies in the rather selfish wish to prove theorem 4.4. It appears that it would be too restrictive to demand for all such $j$ that $\tilde{n}_{j}>0$. Finally, the condition that

$$
\left\{k \mid 1 \leqslant k \leqslant m^{\prime} \wedge \tilde{n}_{k}>0 \wedge k \text { enabled at } p\right\} ; \varnothing
$$

entails that case 2 can never occur twice in succession.

Now for the rest of this subsection let $p \in P$ be fixed. We define a mapping

$$
\Phi: \text { Paths }(\text { Fair }(p)) \rightarrow \text { Paths }(p),
$$

relating to each path $\pi$ in $\operatorname{Fair}(p)$ a fair path in $p$, in exactly the same way as in definition 3.6. We finally repeat theorems 3.7 and 3.8 of the previous section, which together show that the definition of $\operatorname{Fair}(p)$ (using the new definition of $N(\nu, p)$ ) is satisfactory. The former proofs of these theorems have to be altered, as can be seen below.

\section{EXAMPLE}

Consider the alphabet $B=\{0,1,2,3, \cdots\}$ and the process $p \in P$ defined by

$$
p \simeq\{<0, p>,<1, p>,<2, p>, \cdots\}
$$

We give an example of a path $\pi$ in $\operatorname{Fair}(p)$ :

$$
\begin{aligned}
& \pi= \\
& \ll 2^{1}, 1^{1}>, \text { fair }\left(<2^{1}, 1^{1}>, p\right)>, \\
& <0, \text { fair }\left(<1^{1}, 1^{1}>, p\right)>,
\end{aligned}
$$




$$
\begin{aligned}
& <0, \text { fair }\left(<0^{\infty}, 1^{1}>, p\right)>, \\
& <1, \text { fair }\left(<0^{\infty}, 0^{\infty}>, p\right)>, \\
& \ll 13^{1}, 2^{1}, 42^{1}, 1^{1}>, \text { fair }\left(<13^{1}, 2^{1}, 42^{1}, 1^{1}>, p\right)>, \\
& <2, \text { fair }\left(<13^{1}, 2^{1}, 41^{1}, 1^{1}>, p\right)>, \cdots
\end{aligned}
$$

For this $\pi$ we have

$$
\Phi(\pi)=<0, p>,<0, p>,<1, p>,<2, p>, \cdots
$$

THEOREM 4.3

Fair $(p)$ is fair. That is, for all $\pi \in \operatorname{Paths}(\operatorname{Fair}(p)), \pi$ is fair.

\section{PROOF:}

Let $p \in P$ and let $\pi \in P$ aths $($ Fair $(p))$ be such that

$$
\begin{aligned}
\pi & \left.\equiv<a_{1}, q_{1}\right\rangle,\left\langle a_{2}, q_{2}\right\rangle, \ldots \\
& \left.\equiv<a_{1}, p_{1}^{\nu_{1}}\right\rangle,\left\langle a_{2}, p_{2}^{\nu_{2}}\right\rangle, \ldots
\end{aligned}
$$

Suppose $b_{i}$ is enabled infinitely often in $\pi$. We must show that $b_{i}$ occurs infinitely often within $\pi$. From the construction of $\operatorname{Fair}(p)$ it follows that in the sequence $\left(\nu_{j}\right)_{j}$ each index $\nu_{j+1}$ is obtained from $\nu_{j}$ by an application of case 1 or 2 . Since case 1 can be applied only finitely many times in succession, it follows that case 2 must have been applied infinitely many times, each application increasing the length of the index. Therefore there is an $N \in \mathbb{N}$ such that for all $j>N$ :

$$
\text { length }\left(\nu_{j}\right)>i \text {. }
$$

Now we are back in the old situation of the previous section! The proof can be completed as before, but for the new observation that with the increase of the length of an index, only priorities lower than the existing ones are introduced.

\section{TYMORHM 4.4}

Any fair path in $p$ is in the range of the mapping $\Phi$.

\section{PROOF}

Given a fair path $\pi^{\prime} \in \operatorname{Path}(p)$,

$$
\pi^{\prime}=\left\langle b_{i_{1}}, p_{1}\right\rangle,\left\langle b_{i_{2}}, p_{2}\right\rangle, \ldots,
$$

we must construct a path $\pi \in$ Paths $($ Fair $(p))$ such that

$$
\Phi(\pi)=\pi^{\prime}
$$


First, we partition $\mathbb{N}$ into two parts $F$ and $I$, where $F$ is the set of all $i$ such that $b_{i}$ is enabled finitely often (perhaps never) in $\pi^{\prime}$, and $I$ is the set of all $i$ such that $b_{i}$ is enabled infinitely often in $\pi^{\prime}$. Thus:

$$
\mathbb{N}=I \cup F \text {. }
$$

Note that (as in 3.4) for all $i \in F b_{i}$ occurs only finitely often in $\pi^{\prime}$ and for all $i \in I b_{i}$ occurs infinitely often in $\pi^{\prime}$, since $\pi^{\prime}$ is fair. Secondly, we introduce the following functions that will be very useful in our proof.

(a) For all $L \in \mathbb{N}$ we define a (position) function $P o s_{L}: B \rightarrow \mathbb{N}$ by

$$
\operatorname{Pos}_{L}\left(b_{k}\right)=\left[\begin{array}{l}
\text { smallest } L^{\prime} \geqslant L \text { such that } \\
\forall j \geqslant L^{\prime}\left[b_{k} \notin p_{j}\right] \\
\text { smallest } L^{\prime} \geqslant L \text { such that } \\
\exists j\left[L<j<L^{\prime} \wedge b_{i_{j}}=b_{k}\right] \quad \text { if } k \in I .
\end{array}\right.
$$

For $k \in F$ this function gives the smallest position greater than $L$ after which $b_{k}$ is never enabled again. For $k \in I$ the smallest position greater than $L$ is chosen such that $b_{k}$ has occurred (at least) once since $L$.

(b) For all $L, L^{\prime} \in \mathbb{N}$, with $L \leqslant L^{\prime}$, we define a (number) function $\operatorname{Num}_{L, L^{\prime}}: B \rightarrow \mathbb{N}$ by

$$
\operatorname{Num}_{L, L^{\prime}}\left(b_{k}\right)=\left[\begin{array}{c}
1+\quad \text { (number of occurrences in } \pi^{\prime} \\
\text { of } \left.b_{k} \text { between } L \text { and } L^{\prime}\right) \\
\text { (number of occurrences in } \pi^{\prime} \\
\text { of } \left.b_{k} \text { between } L \text { and } L^{\prime}\right)
\end{array} \text { if } k \in F\right.
$$

(In this definition between $L$ and $L^{\prime}$ means including $L$, and excluding $L^{\prime}$.)

We shall define, at each of an infinite sequence of stages $k$, an index $\nu_{k}$ and, corresponding to that index, the $k$-th part of the path $\pi$ corresponding to $\pi^{\prime}$. After we have constructed, at stage $k-1$, the $(k-1)$-th approximation of path $\pi$ corresponding to the initial segment

$$
<b_{i_{1}}, p_{1}>, \ldots,<b_{i_{1}}, p_{l}>
$$

of path $\pi^{\prime}$, then at stage $k$ we shall take into account the basic steps $b_{i_{1},}$ and all the $b_{j}$ 's we have encountered in the preceding stages. We shall make sure that the length of the index $\nu_{k}$ will be, as prescribed by definition 4.2 , strictly bigger than the length of $\nu_{k-1}$. Note that in the previous section, where our alphabet was finite, from the beginning we could focus on all $b_{j}$ 's at the same time.

\section{Stage 1}

For the definition of our first index $\nu_{1}$ we focus on basic step $b_{i_{1}}$. We define

$$
L_{1}=\operatorname{Pos}_{1}\left(b_{i_{1}}\right) \text {, }
$$




$$
\begin{aligned}
& R_{1}=\left\{i_{1}, \ldots, i_{L_{1}-1}\right\}, \\
& M_{1}=\max R_{1} .
\end{aligned}
$$

Our first index $\nu_{1}$, with $\nu_{1}=\left\langle n_{1}^{s_{1}}, \ldots, n_{M_{1}}^{s_{M_{1}}}\right\rangle$, is defined so that

$$
\begin{gathered}
\forall 1 \leqslant j \leqslant M_{1}\left[j \in R_{1} \Rightarrow\left(n_{j}=N u m_{1, L_{1}}\left(b_{j}\right) \wedge s_{j}=1\right) \wedge\right. \\
\left.j \notin R_{1} \Rightarrow\left(n_{j}=0 \wedge s_{j}=\infty\right)\right] .
\end{gathered}
$$

The length of $\nu_{1}$ is $M_{1}$, because according to the definition of indices no holes are allowed in $\nu_{1}$, that is: every index is related to an initial part of the enumeration of our infinite alphabet $\left\{b_{1}, b_{2}, \ldots\right\}$. For those basic steps $b_{j}$ that do not occur in the path $\pi^{\prime}$ before place $L_{1}$, default values $n_{j}=0$ and $s_{j}=\infty$ are chosen in $\nu_{1}$. (Here we use the fact that for newly introduced $j$ 's, $n_{j}$ can get the value 0 once. See the corresponding remark in the explanation following definition 4.2.) With $\nu_{1}$ we can construct the first part of $\pi$ corresponding to the part of $\pi^{\prime}$ before $L_{1}$, starting with $p^{\nu_{1}}$, and repeatedly applying case 1 for the appropriate $b_{i}$, thus decreasing the $n_{i}$ 's until (at step $L_{1}$ ) our index is such that for all $1 \leqslant i \leqslant M_{1}$ :

$$
\begin{aligned}
& \left(i \in F \cap R_{1}\right) \Rightarrow\left(n_{i}=1 \wedge s_{i}=1\right), \\
& \left(i \in I \cap R_{1} \vee i \notin R_{1}\right) \Rightarrow\left(n_{i}=0 \wedge s_{i}=\infty\right) .
\end{aligned}
$$

Now case 2 must be applied, since no $j \in F \cap R_{1}$ is enabled at step $L_{1}$. This brings us to stage 2 .

\section{Stage 2}

We define our next index $\nu_{2}$, taking into account all steps encountered at stage 1 , that is all $b_{i}$ 's with $1 \leqslant i \leqslant M_{1}$, and the next step in the path $\pi^{\prime}$, that is $b_{i_{1}}$. We define

$$
\begin{aligned}
& L_{2}=\max \left(\left\{\operatorname{Pos}_{L_{1}}\left(b_{L_{L_{1}}}\right)\right\} \cup\left\{\operatorname{Pos}_{L_{1}}\left(b_{k}\right) \mid 1 \leqslant k \leqslant M_{1}\right\}\right), \\
& R_{2}=\left\{1, \ldots, M_{1}\right\} \cup\left\{i_{L_{1}}, \ldots, i_{L_{2}-1}\right\}, \\
& M_{2}=1+\max R_{2} .
\end{aligned}
$$

We define our second index $\nu_{2}$, with $\nu_{2}=\left\langle\tilde{n}_{1}^{\tilde{s}_{1}}, \ldots, \tilde{n}_{M_{2}}^{\tilde{s}_{M_{2}}}\right\rangle$, such that

$$
\begin{aligned}
\forall 1 \leqslant j \leqslant M_{2} & {\left[\left(\left(1 \leqslant j \leqslant M_{1} \wedge n_{j}=0\right) \vee\left(j>M_{1} \wedge j \in R_{2}\right) \Rightarrow\right.\right.} \\
& \left.\tilde{n}_{j}=N u m_{L_{1}, L_{2}}\left(b_{j}\right) \wedge \tilde{s}_{j}=1+\max \left\{s_{k} \mid 1 \leqslant k \leqslant M_{1}\right\}\right) \wedge \\
& \left(1 \leqslant j \leqslant M_{1} \wedge n_{j}=1\right) \Rightarrow\left(\tilde{n}_{j}=n_{j} \wedge \tilde{s}_{j}=s_{j}\right) \wedge \\
& \left.\left(j \notin R_{2}\right) \Rightarrow\left(\tilde{n}_{j}=0 \wedge \tilde{s}_{j}=\infty\right)\right] .
\end{aligned}
$$


Note that $M_{2}$, the length of $\nu_{2}$, is strictly bigger than $M_{1}$, the length of $\nu_{1}$. We proceed as before, constructing the part of $\pi$ corresponding to the part of $\pi^{\prime}$ between $L_{1}$ and $L_{2}$. Continuing in this way, we construct a path $\pi$ in fair $(p)$ such that $\Phi(\pi)=\pi^{\prime}$.

\section{INFINITE ITERATION}

Let $P$ be the mathematical domain of section 3, that is, a complete metric space satisfying

$$
P \cong\left\{p_{0}\right\} \cup \mathscr{P}_{c l}(B \times P)
$$

where $B$ is a finite alphabet

$$
B=\left\{b_{1}, \ldots, b_{m}\right\} .
$$

The operation of sequential composition on $P$ is defined in

DEFINITION 5.1 (Sequential composition)

Let $\circ: P \times P \rightarrow P$ be given by

$$
p^{\circ} q=\left[\begin{array}{ll}
q & \text { if } p=p_{0} \\
\left.\left\{<b, p^{\prime} \circ q\right\rangle\left|<b, p^{\prime}\right\rangle \in p\right\} & \text { if } p \neq p_{0}
\end{array}\right.
$$

for all $p$ and $q$ in $P$.

\section{REMARKS}

(1) Because this definition is self-referential, it needs some justification. We observe that $\circ$ can be defined as the unique fixed point of a contraction $\Phi$ of type $\Phi:(P \times P \rightarrow P) \rightarrow(P \times P \rightarrow P)$. (Similar argument to that for Fair: Remark (1) after Definition 3.4.)

(2) It is not very difficult to show that:

$$
\forall p, q, q^{\prime} \in P\left[p \neq p_{0} \Rightarrow d_{P}\left(p^{\circ} q, p^{\circ} q^{\prime}\right) \leqslant \frac{1}{2} d_{P}\left(q, q^{\prime}\right)\right] .
$$

We shall use this property below.

In this section we want to study the operation of infinite iteration of a process $p \in P$. It is defined as follows: 
DEFInITION 5.2 (Infinite iteration)

Let $(\cdots)^{\omega}: P \rightarrow P$ be given by

$$
p^{\omega}=\lim _{n \rightarrow \infty} p^{n}
$$

for $p \in P$, where $p^{0}=p_{0}$ and $p^{n+1}=p^{n} \circ$.

(This limit exists, as can be easily proved using the property of remark (2) above).

Let us now explain how fairness issues come into play by taking the infinite iteration of $p \in P$. Generally, taking the infinite iteration of a process $p \in P$ introduces new infinite paths in $p^{\omega}$ that were not yet present in $p$. When we take, for example, $p=\left\{\left\langle a, p_{0}\right\rangle,\left\langle b, p_{0}\right\rangle\right\}$, then $p$ does not contain any infinite paths, whereas $p^{\omega}$, which satisfies

$$
\left.\left.p^{\omega}=\left\{<a, p^{\omega}\right\rangle,<b, p^{\omega}\right\rangle\right\},
$$

contains many. Some of these are unfair, such as

$$
\pi=\left\langle a, p^{\omega}\right\rangle,\left\langle a, p^{\omega}\right\rangle,\left\langle a, p^{\omega}\right\rangle, \ldots,
$$

which is unfair with respect to $b$. Such unfair paths $\pi$ we call globally unfair. We do not call every unfair path in $p^{\omega}$ globally unfair, only those that are introduced, so to speak, by taking the infinite iteration of $p$. Another example may illustrate this point. (Formal definitions follow below.) Consider a process $p \in P$ satisfying

$$
\left.\left.p=\{<a, p\rangle,<b, p_{0}\right\rangle\right\} .
$$

Then $p^{\omega}$ will contain the unfair paths

$$
\begin{aligned}
& <a, p>,<a, p>, \ldots \text {, } \\
& \langle b, p\rangle,\langle a, p\rangle,\langle a, p\rangle, \ldots, \\
& \langle b, p\rangle,\langle b, p\rangle,\langle a, p\rangle,\langle a, p\rangle, \ldots \text {, etc. }
\end{aligned}
$$

The unfairness of these paths is, as it were, reducible to the unfairness of the path

$$
<a, p>,<a, p>, \ldots,
$$

which was already present in $p$. Therefore they will not be called globally unfair paths.

There is a second notion of unfairness, which plays a role here. It is called node (or local) unfairness. Again we explain it here by giving an example, the formal definition following below. Let $p \in P$ contain the node $p^{\prime}=\left\{\left\langle a, p_{1}\right\rangle,\left\langle b, p_{2}\right\rangle\right\}$. Let $\pi \in \boldsymbol{P a t h s}\left(p^{\omega}\right)$ and suppose $\pi$ passes through $p^{\prime}$ infinitely many times. If it is the case that in $\pi$ the next step that is taken after passing through $p^{\prime}$ is always $a$, and never $b$, 
we call $\pi$ node unfair (with respect to the node $p^{\prime}$ ). The reason for this terminology is obvious: although $b$ is infinitely often enabled in $\pi$ at node $p^{\prime}$, it is never chosen in $\pi$ as the next step after $p^{\prime}$.

The notions of global and node unfairness are in a sense independent. Let $p \in P$ be given by

$$
\begin{aligned}
& p=\left\{\left\langle b, p^{\prime}\right\rangle\right\}, \text { where } \\
& p^{\prime}=\left\{\langle a, p\rangle,\left\langle b, p_{0}\right\rangle\right\} .
\end{aligned}
$$

Consider $\pi \in P$ aths $\left(p^{\omega}\right)$, given by

$$
\pi=\left\langle b, p^{\prime}\right\rangle,\langle a, p\rangle,\left\langle b, p^{\prime}\right\rangle,\langle a, p\rangle, \ldots
$$

This path is not globally unfair, but is node unfair with respect to the node $p^{\prime}$. Thus node unfairness does not imply global unfairness. The same holds in the opposite direction. Let $p \in P$ be defined by

$$
\left.p=\left\{\left\langle a^{n},\left\{<a, p_{0}\right\rangle,\left\langle b, p_{0}\right\rangle\right\}\right\rangle \mid n \in \mathbb{N}\right\} \cup\left\{a^{\omega}\right\},
$$

using $a^{n}$ and $a^{\omega}$ as shorthand with an obvious interpretation. (The fact that $a^{\omega} \in p$ is not important for the point we want to make with this example, but is implied by the (topological) closedness of $p$.) Now it is not difficult to find a path

$$
\pi=\left\langle a, p_{1}\right\rangle,\left\langle a, p_{2}\right\rangle,\left\langle a, p_{3}\right\rangle, \ldots
$$

in $\operatorname{Paths}\left(p^{\omega}\right)$ (with $p_{1}, p_{2}, p_{3}, \ldots$ nodes of $p$ ) that is globally unfair (with respect to $b$ ), but fair with respect to every node of $p$, although it passes through $p$ infinitely many times.

Note that the notions of global and node fairness are not related to each other as those of "top level" and "all levels" fairness (cf. Section 3.3 in [F]), since "all levels" fairness implies "top level" fairness, but (as we have seen) neither of global and node fairness implies the other.

Let us now proceed with formally defining these notions of global and node unfairness. Actually, we shall define what we consider to be globally fair and node fair. For this we need the following notion.

DEFINITION 5.3 (Iteration paths)

Let $p \in P, \pi \in \boldsymbol{P a t h s}\left(p^{\omega}\right)$. We call $\pi$ an (infinite) iteration path, whenever $\pi$ is the concatenation of an infinite sequence of finite paths $\pi_{1}, \pi_{2}, \ldots \in P$ aths $(p)$ :

$$
\pi=\pi_{1}^{\circ} \pi_{2} \circ \pi_{3} \circ \ldots
$$

For a basic step $b$ occurring in $\pi_{k}$ we say that $b$ occurs in the $k$-th instantiation of $p$. 
DEFINITION 5.4 (Global fairness)

Let $p \in P, \pi \in P$ aths $\left(p^{\omega}\right)$. We call $\pi$ globally fair whenever

(1) $\pi$ is fair (in the sense of definition 2.3); or

(2) $\pi$ is not an iteration path.

We call $p^{\omega}$ globally fair whenever all paths in $p^{\omega}$ are globally fair.

REMARK: It follows that a path in $p^{\omega}$ is globally unfair if and only if it is an iteration path and unfair.

\section{DEFINITION 5.5 (Node fairness)}

Let $p \in P, \pi \in \boldsymbol{P a t h s}\left(p^{\omega}\right)$. We call $\pi$ node fair with respect to $p^{\prime}$, for a subnode $p^{\prime}$ of $p$, whenever it is the case that: if $\pi$ passes through $p^{\prime}$ infinitely often, then for all $b \in B$ that are enabled in $p^{\prime}: b$ occurs infinitely often in $\pi$, immediately after $p^{\prime}$. We call $\pi$ node fair if it is node fair with respect to every subnode $p^{\prime}$ of $p$. Finally we call $p^{\omega}$ node fair if all paths in Paths $\left(p^{\omega}\right)$ are node fair.

The aim of this section is to define two fair versions of the infinite iteration operator:

$$
(\cdots)^{\omega_{\text {far }}}: p \rightarrow p
$$

such that the result $p^{\omega_{\text {jar }}}$ will be globally fair and node fair respectively. For this purpose we first give an alternative definition of infinite iteration, which will be used as a starting point for defining $(\cdots)^{\omega_{\text {far }}}$.

PROPOSITION 5.6 (Alternative definition of infinite iteration)

Let $p \in P$. We define App $p: P \rightarrow P$ by

$$
\begin{aligned}
& \operatorname{App}_{p}\left(p_{0}\right)=p \circ \operatorname{App}_{p}(p) \\
& \operatorname{App}_{p}(q)=\left\{<a, \operatorname{App}_{p}\left(q^{\prime}\right)>\mid<a, q^{\prime}>\in q\right\}, \text { if } q \neq p_{0} .
\end{aligned}
$$

(Read "append" for App.) Then we have:

$$
p^{\omega}=\operatorname{App}_{p}(p)
$$

RFMAKKS

(1) Formally, $A p p_{p}$ can be defined as the unique fixed point of the function $\Phi_{p}:(P \rightarrow P) \rightarrow(P \rightarrow P)$, given by

$$
\begin{aligned}
& \Phi_{p}(\phi)\left(p_{0}\right)=p^{\circ} \phi(p), \\
& \Phi_{p}(\phi)(q)=\left\{<a, \phi\left(q^{\prime}\right)>\mid<a, q^{\prime}>\in q\right\}, \text { if } q \neq p_{0} .
\end{aligned}
$$

(Again it can be shown that $\Phi_{p}$ is contracting by a similar argument as given in 
Remark (1) following Definition 3.4)

(2) The function $A p p_{p}$ applied to an argument $q \in P$ replaces all occurrences of $p_{0}$ in $q$ by $p$, in which, recursively, all occurrences of $p_{0}$ are again replaced by $p$.

(3) From proposition 5.6 it follows that $\operatorname{App}_{p}\left(p_{0}\right)=\operatorname{App}_{p}(p)$.

Proof of THE Proposition

We define, for fixed $p \in P$, a function $\phi_{p}: P \rightarrow P$ by

$$
\phi_{p}(q)=q \circ p^{\omega} .
$$

We have

$$
\begin{aligned}
\phi_{p}\left(p_{0}\right) & =p_{0} \circ p^{\omega}=p^{\omega}=p^{\circ} p^{\omega} \\
& =p^{\circ}\left(p^{\circ} p^{\omega}\right)=p^{\circ} \phi_{p}(p)
\end{aligned}
$$

and, for $q \in P, q \neq p_{0}$ :

$$
\begin{aligned}
\phi_{p}(q) & \left.=q^{\circ} p^{\omega} \quad \text { (definition of } \circ\right) \\
& =\left\{<a, q^{\prime} \circ p^{\omega}>\mid<a, q^{\prime}>\in q\right\} \\
& =\left\{<a, \phi_{p}\left(q^{\prime}\right)>\mid<a, q^{\prime}>\in q\right\} .
\end{aligned}
$$

From this it follows that $\phi_{p}$ is also a fixed point of $\Phi_{p}$. Because $\Phi_{p}$ is contracting, it has a unique fixed point, thus $\phi_{p}=\operatorname{App}_{p}$. Thus

$$
p^{\omega}=p \circ p^{\omega}=\phi_{p}(p)=\operatorname{App}_{p}(p) .
$$

(1) Global faimess

In this subsection we set out to define a fair version

$$
(\cdots)^{\omega_{\text {fair }}}: P \rightarrow P^{F I n d}
$$

of the operation of infinite iteration such, that for $p$ in $P$ the result $p^{\omega_{\text {fair }}}$ will be globally fair. The range $P^{F I n d}$ of this mapping $(\cdots)^{\omega_{\text {fair }}}$ is given by

$$
P^{F I n d}=\left\{p_{0}\right\} \cup \mathscr{P}_{c l}\left(A \times P^{F I n d}\right),
$$

with

$$
A=B \cup \text { FIndex, }
$$

where FIndex is a set of indices to be defined below. A naive first attempt would be to define

$$
p^{\omega_{\text {far }}}=\operatorname{Fair}\left(p^{\omega}\right)
$$


with the function Fair as in definition 3.4. This would be wrong, according to our definition of global fairness. The function Fair transforms its argument into a process, in which all unfair paths have disappeared. However, not every unfair path in $p^{\omega}$ is globally unfair, only those that are iteration paths. Thus the function Fair removes too many paths from $p^{\omega}$. (For an illustration see the informal explanation above.) Therefore we have to come up with another solution. We shall use the definition of $p^{\omega}$ as $\operatorname{App}_{p}(p)$ as a starting point for the definition of $p^{\omega_{\text {far }}}$, but changing it by again using indices (as we did in the definition of Fair) to label the nodes of $p$. After having defined $p^{\omega_{\text {fair }}}$, we shall clarify the relation between $p^{\omega_{f a r}}$ and $p^{\omega}$ by defining a mapping

$$
\Phi: \operatorname{Paths}\left(p^{\omega_{\text {fair }}}\right) \rightarrow \text { Paths }\left(p^{\omega}\right) .
$$

Although the idea of defining $p^{\omega_{\text {far }}}$ as $\boldsymbol{F a i r}\left(p^{\omega}\right)$ does not work (as was mentioned above), the definition of $(\cdots)^{\omega_{\text {far }}}$ will be surprisingly similar to that of the function Fair. The reason is the following: in constructing $p^{\omega_{\text {fair }}}$ for a given $p \in P$, we do two things at the same time. On the one hand we construct (a special version of) the infinite iteration of $p$, and on the other hand we select certain paths, namely those that are globally fair. The first task is performed along the lines of the definition of $A p p_{p}$, the second task is realised following the definition of Fair. So in some sense the definition of $p^{\omega_{\text {fair }}}$ will be a combination of the definitions of $A_{p p}$ and Fair (see proposition 5.6 and definition 3.4 ).

Definitron 5.7 (Flag indices). The set of flag indices, with typical element $\mu$, is defined by:

$$
\begin{aligned}
& \text { FIndex }= \\
& \left\{<<n_{1}, s_{1}, f_{1}>, \ldots,<n_{m}, s_{m}, f_{m} \gg \mid n_{i} \geqslant 0,0 \leqslant s_{i} \leqslant \infty, f_{i} \in\{U, D\}\right\}
\end{aligned}
$$

where $m$ is the number of basic steps in our finite alphabet $B$, and $\{U, D\}$ is the set of flags, containing two elements: $U$ (for "up") and $D$ (for "down").

The interpretation of $n_{i}$ and $s_{i}$ is as in definition 3.1 (see the informal explanation that follows there), but for the difference that only the first occurrence of $b_{i}$ in each instantiation of $p$ in $p^{\omega_{\text {fair }}}$ will cause $n_{i}$ to be decreased by 1 . Whether or not $b_{i}$ has been chosen in a given instantiation of $p$, is indicated by the flag $f_{i}$. If it is up, $b_{i}$ has not yet been chosen, and if it is down, $b_{i}$ has been chosen at least once in the current instantiation of $p$.

We need the following operations on indices.

DeFINITION 5.8 
Let $\mu \in$ FIndex, with $\mu=<<n_{1}, s_{1}, f_{1}>, \ldots,<n_{m}, s_{m}, f_{m} \gg$, and let $i \in\{1, \ldots, m\}$. We define

$\mu^{-}[i]=\left[\begin{array}{ll}\mu & \text { if } f_{i}=D \\ <<n_{1}, s_{1}, f_{1}>, \ldots,<n_{i}-1, s_{i}, D>, \ldots,<n_{m}, s_{m}, f_{m}>> & \text { if } f_{i}=U \wedge n_{i}>1 \\ <<n_{1}, s_{1}, f_{1}>, \ldots,<0, \infty, D>, \ldots,<n_{m}, s_{m}, f_{m} \gg & \text { if } f_{i}=U \wedge n_{i}=1 \\ \text { undefined } & \text { otherwise. }\end{array}\right.$

For $\mu \in \boldsymbol{F I n d e x}$ with $f_{i}=U$ the interpretation of $\mu^{-}[i]$ is as in definition 3.2, with the difference that $U$ is changed to $D$. This indicates that in the current instantiation of $p$ the basic step $b_{i}$ has been chosen (at least once). If $f_{i}=D$, then $\mu^{-}[i]=\mu$, as indicated above. This will be explained below, after the definition of $p^{\omega_{\text {fair }}}$.

\section{Definition 5.9}

Let $\mu \in \boldsymbol{F I n d e x}$, with $\mu=<<n_{1}, s_{1}, f_{1}>, \ldots,<n_{m}, s_{m}, f_{m} \gg$. We define

$$
\begin{aligned}
N(\mu)= & \left\{<<\tilde{n}_{1}, \tilde{s}_{1}, f_{1}>, \ldots,<\tilde{n}_{m}, \tilde{s}_{m}, f_{m}>1\right. \\
& \forall i \in\{1, \ldots, m\}\left[\left(n_{i}=0 \wedge s_{i}=\infty \Rightarrow \tilde{n}_{i}>0 \wedge \tilde{s}_{i}=1+\max \left\{s_{j} \mid 1 \leqslant j \leqslant m\right\}\right)\right. \\
& \left.\wedge\left(n_{i}>0 \wedge s_{i}<\infty \Rightarrow \tilde{n}_{i}=n_{i} \wedge \tilde{s}_{i}=s_{i}\right)\right\} .
\end{aligned}
$$

The definition of $N(\mu)$ is as in definition 3.3, because the flags do not matter here.

Definition 5.10

Let $\mu \in$ FIndex, with $\left.\mu=<<n_{1}, s_{1}, f_{1}\right\rangle, \ldots,<n_{m}, s_{m}, f_{m} \gg$. Then

$$
\mu^{U}=\ll<n_{1}, s_{1}, U>, \ldots,<n_{m}, s_{m}, U>>\text {. }
$$

This operation sets all flags to "up" and is used upon entrance to a new instantiation of $p$. Now we are ready to define $(\cdots)^{\omega_{\text {far }}}$.

DeFINITION 5.11 (Fair infinite iteration)

We define $(\cdots)^{\omega_{\text {fair }}}: P \rightarrow P^{\text {FInd }}$. Let $p \in P$. Then

$$
p^{\omega_{\text {fair }}}=\sum_{\mu \in I_{0}} \operatorname{App}_{p}(p, \mu)
$$

where

$$
I_{0}=\left\{\ll<n_{1}, 1, U>, \ldots,<n_{m}, 1, U>>\mid n_{i}>0\right\}
$$

and for given $p \in P$

$$
\operatorname{App}_{p}: P \times \text { FIndex } \rightarrow P^{F I n d}
$$

is defined as follows. (We write $q^{\mu}$ for $\operatorname{App}_{p}(q, \mu)$.) Let $\mu \in \boldsymbol{F I n d e x}$. We define 


$$
\operatorname{App}_{p}\left(p_{0}, \mu\right)=\operatorname{App}_{p}\left(p, \mu^{U}\right)
$$

For $q \in P, q \neq p_{0}$, we distinguish two cases.

Case 1:

$$
\begin{aligned}
& \text { If } \exists i \in\{1, \ldots, m\}\left[\operatorname{enabled}(i) \wedge\left(f_{i}=D \vee\left(s_{i}<\infty \wedge n_{i}>0\right)\right)\right], \\
& \text { then } q^{\mu}=\left\{<b_{i}, \bar{q}^{\mu^{-}[i]}>\mid<b_{i}, \bar{q}>\in q \wedge\right. \\
& \left.\qquad\left(f_{i}=D \vee\left(s_{i}<\infty \wedge n_{i}>0 \wedge s_{i}=\min \left\{s_{1}, \ldots, s_{m}\right\}\right)\right)\right\} .
\end{aligned}
$$

Case 2:

$$
\begin{aligned}
& \text { If } \forall i \in\{1, \ldots, m\}\left[\operatorname{enabled}(i) \Rightarrow\left(f_{i}=U \wedge s_{i}=\infty \wedge n_{i}=0\right)\right] \\
& \text { then } q^{\mu}=\sum_{\mu^{\prime} \in N(\mu)} q^{\mu^{\prime}} \text {. }
\end{aligned}
$$

\section{REMARKS}

(1) The remarks (1), (2), and (3) following definition 3.4 apply also to the above definition.

(2) We give some informal explanation of this definition by referring to remark (4) after definition 3.4 and making explicit what is different here. First, when we reach $p_{0}$ in the definition (3.4) of fair, we are done: $\operatorname{fair}\left(p_{0}, \nu\right)=p_{0}$. Here we continue by appending $p$ to $p_{0}$, together with the index $\mu$ changed into $\mu^{U}: \operatorname{App}_{p}\left(p_{0}, \mu\right)=\operatorname{App}_{p}\left(p, \mu^{U}\right)$. The reason why we append $p$ to $p_{0}$ is obvious: we are building the infinite iteration of $p$. (See proposition 5.6.) The index $\mu$ is changed to $\mu^{U}$, that is all flags $f_{i}$ of $\mu$ are set to $U$ to indicate the entrance of a new instantiation of $p$. The second important difference between this definition and definition 3.4 is the role played by the flags. Let $q \in P$ with $\left\langle b_{i}, \bar{q}\right\rangle \in q$ for some $\bar{q} \in P, b_{i} \in B$. If $f_{i}=D$ (down), then $b_{i}$ has already been chosen (at least once) in the current instantiation of $p$. Therefore it may be chosen unrestrictedly, even infinitely many times, within this instantiation of $p$ (no matter what the values of $n_{i}$ and $s_{i}$ are). In this case we have: $\mu^{-}[i]=\mu$, formally expressing that $b_{i}$ may pass "for free" without changing the values of $n_{i}$ and $s_{i}$. The reason for letting $b_{i}$ pass for free is that it provides us with the presence within $p \omega_{\text {far }}^{\omega}$ of those infinite paths (possibly unfair) that are not iteration paths (and, hence, not globally unfair). If on the other hand $f_{i}=U$ and $n_{i}>0$ and $s_{i}=\min \left\{s_{1}, \ldots, s_{m}\right\}<\infty$, then $b_{i}$ may be chosen (as in case 2 of definition 3.4), but now $\mu$ is changed into $\mu^{-}[i]$ by changing the values of $n_{i}$ and $s_{i}$ (as in definition 3.4) and by changing the flag $f_{i}$ to $D$.

Now for the rest of this subsection let $p \in P$ be fixed. We define a mapping 


\section{$\Phi: \operatorname{Paths}\left(p^{\omega_{\text {far }}}\right) \rightarrow \operatorname{Paths}\left(p^{\omega}\right)$,}

relating to each iteration path in $p^{\omega_{\text {far }}}$ a corresponding fair iteration path in $p^{\omega}$. We start by re-stating lemma 3.5 .

LEMMA 5.12

Let $\bar{p} \in P$, with $\bar{p}=p_{0}, \mu \in$ FIndex, and $\left\langle a, q>\in A p p_{p}(\bar{p}, \mu)\right.$ for $a \in B$ and $q \in P$. Then there exist $P^{\prime} \in P$ and $\mu^{\prime} \in$ FIndex such that

$$
\begin{aligned}
& q=\operatorname{App}_{p}\left(p^{\prime}, \mu^{\prime}\right) \wedge \\
& a \in \text { FIndex } \Rightarrow p^{\prime}=\bar{p} \wedge \\
& a \in B \Rightarrow<a, p^{\prime}>\in \bar{p} .
\end{aligned}
$$

The proof is straightforward from the definition of $\bar{p}^{\mu}\left(=A p p_{p}(\bar{p}, \mu)\right)$.

Definition 5.13 (The mapping $\Phi$ )

Let

$$
\pi=\left\langle a_{0}, q_{0}>,<a_{1}, q_{1}>, \ldots\right.
$$

be a path in $p^{\omega_{\text {fair }}}$. We can rewrite it as:

$$
\pi=\left\langle a_{0}, p^{\mu}\right\rangle,\left\langle a_{1}, p_{1}^{\mu_{1}}\right\rangle,\left\langle a_{2}, p_{2}^{\mu_{2}}\right\rangle, \ldots
$$

for certain $\mu_{1} \mu_{1}, \mu_{2}, \ldots \in F$ Index and $p_{1}, p_{2}, \ldots \in P$. If we omit in $\pi$ all pairs $\left\langle a_{i}, p_{i}^{\mu_{i}}\right\rangle$ with $a_{i} \in F I n d e x$, and further all superscripts $\mu_{i}$, we get a sequence

$$
\Phi(\pi)=<a_{i_{1}}, p_{i_{1}}>,<a_{i_{2}}, p_{i_{2}}>, \ldots
$$

which is a path in $p^{\omega}$. We call $\Phi(\pi)$ the path in $p$ corresponding to the path $\pi$ in $p^{\omega_{\text {far }}}$. This defines a mapping

$$
\Phi: \text { Paths }\left(p^{\omega_{\text {fair }}}\right) \rightarrow \text { Paths }\left(p^{\omega}\right) .
$$

\section{THEOREM 5.14}

$p^{\omega_{\text {fair }}}$ is globally fair. That is, for all $\pi \in P$ aths $\left(p^{\omega_{\text {far }}}\right)$, if $\pi$ is an iteration path, then $\pi$ is fair.

ProOF

Let $\pi \in \operatorname{Paths}\left(p^{\omega_{f a r}}\right)$ and suppose $\pi$ is an iteration path. We reduce the proof of this theorem to that of theorem 3.7 by making the following observation. Since $\pi$ is an 
infinite iteration path it enters infinitely often into a new instantiation of $p$. Upon each entrance, all flags are raised (set to "up"). As was observed above, if $f_{i}=U$ (for $i \in\{1, \ldots, m\})$, then $b_{i}$ is treated in case 1 of definition 5.11 above in exactly the same way as in case 1 of definition 3.4. Because this situation arises infinitely often, the argument given in the proof of theorem 3.7 also applies here. (Note that case 2 in both definitions 3.4 and 5.11 is the same.)

REMARK: Formally we have to extend definition 5.4 of global fairness to processes in $P^{F I n d}$. This can be done straightforwardly.

I'HYorim 5.15: Any globally fair path in $p^{\omega}$ is in the range of the mapping $\Phi$.

\section{PROOF}

Let $\pi^{\prime} \in \boldsymbol{P a t h s}\left(p^{\omega}\right)$ such that $\pi^{\prime}$ is globally fair. We must construct a path $\pi \in P a t h s\left(p^{\omega_{\text {far }}}\right)$ such that

$$
\Phi(\pi)=\pi^{\prime} .
$$

We distinguish between two cases: first, that $\pi^{\prime}$ is not an iteration path (and possibly unfair); second, that $\pi^{\prime}$ is an iteration path and fair.

(1) Suppose $\pi^{\prime}$ is not an iteration path. Without loss of generality we may assume that $\pi^{\prime}$ lies entirely within $p$ (that is, the first instantiation of $p$ in $p^{\omega}$ ). We define a flag index $\mu$ by

$$
\mu=<<1,1, U>, \ldots,<1,1, U>>
$$

and take $\left\langle\mu, p^{\mu}\right\rangle$ as the first element of the path $\pi$ that we are constructing. Now we can continue the construction of $\pi$ by repeatedly applying case 1 (of definition 5.11) for the appropriate $b_{i}^{\prime}$ 's (namely, those that occur in $\pi^{\prime}$ ). Each time we encounter a $b_{i}$ for the first time, the corresponding triple $\langle 1,1, U\rangle$ in the index is changed into $\langle 0, \infty, D\rangle$. From this moment on $b_{i}$ may be chosen unrestrictedly within this instantiation of $p$ (in which the path $\pi^{\prime}$ lies), without changing the index. The path $\pi$ thus constructed is an element of Paths ( $\left.p^{\omega_{\text {far }}}\right)$. Furthermore: $\Phi(\pi)=\pi^{\prime}$. (Note that it is of no importance whether $\pi^{\prime}$ is fair or not.)

(2) Suppose $\pi^{\prime}$ is a fair infinite iteration path. As in the proof of theorem 5.14 , we reduce this proof to that of the corresponding theorem in section 3 (theorem 3.8) by observing that the latter only needs a slight modification. When we count the number of times that a certain $b_{i}$ occurs before a given step $l_{j}$ in the path $\pi^{\prime}$, we have to count only the first occurrences of $b_{i}$ in different instantiations of $p$. With this change in mind the proof of 3.8 can easily be transformed into a proof of this theorem. 
(2) Node fairness

Let us now forget about global fairness and focus on the second notion: node fairness. We again set out to define a fair version

$$
(\cdots)^{\omega_{\text {far }}}: P \rightarrow P^{N I n d}
$$

of the operation of infinite iteration but now such that for all $p \in P$ the result $p^{\omega_{\text {fair }}}$ will be node fair. The domain $P^{\text {NInd }}$ is like $P^{\text {Ind }}$ and $P^{\text {FInd }}$, but with

$$
A=B \cup N \text { Index, }
$$

with NIndex a set of indices to be defined below.

In constructing this second version of infinite iteration we proceed globally as in the previous subsection, now using node indices in order to ensure the node fairness of $p^{\omega_{\text {fair }}}$, instead of flag indices, which were used above. We shall characterize (and even identify) a subnode of a given process $p \in P$ by the subpath in $p$ that leads to it.

\section{Definition 5.16 (Nodes)}

Let $p \in P$. We define the set of nodes of $p$ by

$$
\operatorname{Nodes}(p)=\left\{\pi \mid \exists \pi^{\prime} \in \boldsymbol{P a t h s}(p)\left[\pi \text { is a finite prefix of } \pi^{\prime}\right]\right\} .
$$

For $\pi \in \operatorname{Nodes}(p)$, with $\left.\left.\pi=<a_{1}, p_{1}\right\rangle, \ldots,<a_{n}, p_{n}\right\rangle$, we define

$$
\operatorname{end}(\pi)=p_{n} \text {. }
$$

(When no confusion is possible we sometimes identify $\pi$ and end $(\pi)$.) We set end $(\epsilon)=p$, where $\epsilon$ is the empty path

The set of node indices for a given $p \in P$ is defined as follows. Each node index for $p$ has two components: the first is a finite mapping, associating with each of a (finite) set of nodes of $p$ an index as defined in 3.1; and the second is a node of $p$. Such a node index schedules the fairness of paths with respect to this second component. At each moment in the construction of $p^{\omega_{\text {farr }}}$, we consider only a finite number of nodes (the domain of the first component), namely those that we have encountered thus far.

Definition 5.17 (Node indices)

Let $p \in P$. We define the set of node indices for $p$ as follows:

$$
\operatorname{NIndex} x_{p}\left(\operatorname{Nodes}(p) \rightarrow{ }^{\text {fin }} \text { Index }\right) \times \operatorname{Nodes}(p),
$$

where $\rightarrow{ }^{f i n}$ denotes the set of partial functions on $\operatorname{Nodes}(p)$ with a finite domain, and Index is defined as in definition 3.1. A typical element of NIndex is denoted $\rho=\left(\rho_{1}, \rho_{2}\right)$. For $\rho_{1} \in \operatorname{Nodes}(p) \rightarrow{ }^{\text {fin }}$ Index we use the variant notation for functions: 
for $\pi, \pi^{\prime} \in \operatorname{Nodes}(p)$ and $\nu \in \operatorname{Index}$,

$$
\rho_{1}\{\nu / \pi\}(\pi)=\left[\begin{array}{ll}
\nu & \text { if } \pi=\bar{\pi} \\
\rho_{1}(\bar{\pi}) & \text { if } \pi \neq \bar{\pi} .
\end{array}\right.
$$

(We shall use this notation whether or not $\pi \in \operatorname{domain}(\rho)$.)

We again need the operations $\nu^{-}[i]$ and $N(\nu)$ on indices $\nu \in$ Index (see definitions 3.2 and 3.3). They are used in the following

DEFINITION 5.18 (Fair infinite iteration)

We define $(\cdots)^{\omega_{\text {fair }}}: P \rightarrow P^{N I n d}$. Let $p \in P$. Then

$$
p^{\omega_{f a i r}}=\sum_{\rho_{1} \in\{\epsilon\} \rightarrow I_{0}} \operatorname{App}_{p}\left(p,\left(\rho_{1}, \epsilon\right)\right)
$$

where $\epsilon$ is the empty subpath of $p$ (identifying $p$ as a subnode of itself),

$$
\left.I_{0}=\left\{<n_{1}^{1}, \ldots, n_{m}^{1}\right\rangle \mid n_{i}>0\right\}
$$

and for given $p \in P$

$$
\text { App }_{p}: P \times N \text { Index } \rightarrow P^{N I n d}
$$

is defined as follows. (We write $q^{\rho}$ for $\boldsymbol{A p p}_{p}(q, \rho)$.) Let $\rho \in$ NIndex, $\rho=\left(\rho_{1}, \rho_{2}\right)$ and let $q \in P$. If $q \neq \operatorname{end}\left(\rho_{2}\right)$, then $\operatorname{App}_{p}(q, \rho)$ is undefined. Now suppose that $q=\boldsymbol{e n d}\left(\rho_{2}\right)$. Then we define

$$
\operatorname{App}_{p}\left(p_{0}, \rho\right)=\operatorname{App}_{p}\left(p_{0},\left(\rho_{1}, \epsilon\right)\right) .
$$

For $q \neq p_{0}$ we distinguish two cases.

Case $(a): \rho_{2} \in \operatorname{domain}\left(\rho_{1}\right)$. Let $\rho_{1}\left(\rho_{2}\right)=\nu=\left\langle n_{1}^{s_{1}}, \ldots, n_{m}^{s_{m}}\right\rangle$. Then

(a 1) If $\exists i \in\{1, \ldots, m\}$ [enabled $\left.(i) \wedge n_{i}>0 \wedge s_{i}<\infty\right]$, then

$q^{\rho}=\left\{<b_{i}, \bar{q}^{\left(\rho_{1}\left\{\nu^{-}[i] / \rho_{2}\right\}, \rho_{2} \circ<b_{i}, \bar{q}>\right)}>\mid<b_{i}, \bar{q}>\in q \wedge s_{i}=\min \left\{s_{j} \mid 1 \leqslant j \leqslant m\right\}\right\}$.

(a2) If $\forall i \in\{1, \ldots, m\}\left[\operatorname{enabled}(i) \Rightarrow n_{i}=0 \wedge s_{i}=\infty\right]$, then

$$
q^{\rho}=\sum_{\nu^{\prime} \in N(\nu)} q^{\left(\rho_{1}\left\{\nu^{\prime} / \rho_{2}\right\}, \rho_{2}\right)}
$$

Case $(b): \rho_{2} \notin \operatorname{domain}\left(\rho_{1}\right)$. Then

$$
q^{\rho}=\sum_{\nu^{\prime} \in I_{0}} q^{\left(\rho_{1}\left\{\nu^{\prime} / \rho_{2}\right\}, \rho_{2}\right)}
$$

where $I_{0}$ is as above. 


\section{REMARKS}

(1) The remarks (1), (2), and (3) following definition 3.4 apply also to the above definition.

(2) We have that $\operatorname{App}_{p}(q, \rho)$ is undefined whenever $q \neq \operatorname{end}\left(\rho_{2}\right)$. This implies (since $\left.p_{2} \in \operatorname{Nodes}(p)\right)$ that $A p p_{p}$ is defined on nodes of $p$ only, which seems quite natural.

(3) We give some informal explanation of the definition above. If we arrive at $p_{0}$, with index $p$, we continue with $\operatorname{App}_{p}\left(p, \rho^{\prime}\right)$. Here $\rho^{\prime}=\left(\rho_{1}, \epsilon\right)$, that is, the second component of $\rho^{\prime}$ now indicates that the node we are treating next is $(e n d(\epsilon)=) p$ itself. The interpretation of cases $(a 1)$ and $(a 2)$ above is entirely similar to that of the cases 1 and 2 in definition 3.4: if $\rho_{2} \in \operatorname{domain}\left(\rho_{1}\right)$, then $v=\rho_{1}\left(\rho_{2}\right)$ is treated exactly as before. A small difference is that, in (a1), the second component $\rho_{2}$ is extended with $\left\langle b_{i}, \bar{q}\right\rangle$ to denote that the next node of $p$ that is treated is $\bar{q}$ $\left(=\operatorname{end}\left(\rho_{2} \circ<b_{i}, \bar{q}>\right)\right.$ ). If $\rho_{2} \notin \operatorname{domain}\left(\rho_{1}\right)$, an extension of the domain of $\rho_{1}$ takes place. Here $I_{0}$ is the set of initial indices (as in definition 3.4).

Now for the rest of this subsection let $p \in P$ be fixed. As in definitions 3.6 and 5.13 we can define a mapping

$$
\Phi: \operatorname{Paths}\left(p^{\omega_{\text {fair }}}\right) \rightarrow \operatorname{Paths}\left(p^{\omega}\right) .
$$

The following two theorems can be proved by easy generalizations of the corresponding proofs (3.7 and 3.8) in section 3.

THEOREM $5.19 p^{\omega_{\text {fair }}}$ is node fair.

THEOREM 5.20 Any node fair path in $p^{\omega}$ is in the range of the mapping $\Phi$.

Combining global and node fairness

We could now combine the two definitions (5.11 and 5.18) of fair infinite iteration and construct a function

$$
(\cdots)^{\omega_{\text {fair }}}: P \rightarrow P
$$

such that $p^{\omega_{\text {fair }}}$ would be both globally and node fair. We do not do this and confine ourselves to the observation that it would be a straightforward and dull exercise. Similarly for the generalization to an infinite alphabet. 
6. REFERENCES

[ABKR] P. America, J.W. De BAKker, J.N. KoK, J.J.M.M. Rutten, $A$ denotational semantics for a parallel object-oriented language, Information and Computation Vol. 83 (No. 2), 1989, pp. 152-205.

[AO] K.R. APT, E.-R. Olderog, Proof rules dealing with fairness, Science of Computer Programming 3, 1983, pp. 65-100.

[AR] P. AMERICA, J.J.M.M. RutTen, Solving reflexive domain equations in a category of complete metric spaces, Journal of Computer and System Sciences Vol. 39 (No. 3), 1989, pp. 343-375.

[BK] J.A. Bergstra, J.W. Klop, A convergence theorem in process algebra, Report CS-R8733, Centre for Mathematics and Computer Science, Amsterdam, Netherlands, 1987.

[BZ1] J.W. DE BAKKer, J.I. ZuCKer, Processes and the denotational semantics of concurrency, Information and Control 54, 1982, pp. 70-120.

[BZ2] J.W. DE BAKker, J.I. Zucker, Processes and a fair semantics for the $A D A$ rendez-vous, in: Proceedings 10th ICALP (J. Diaz, Ed.) Lecture Notes in Computer Science 154, Springer-Verlag, 1983, pp. 52-66.

[BZ3] J.W. DE BAKKER, J.I. ZUCKER, Compactness in semantics for merge and fair merge, Proceedings Workshop Logics of Programs, (E. Clarke \& D. Kozen, Eds.) Pittsburgh, Lecture Notes in Computer Science 164 Springer-Verlag, 1983, pp. 18-33.

[C] W.D. Clinger, Foundations of actor semantics, Ph. D. thesis, Massachusetts Institute of Technology (AI-TR-633), 1981.

[CS] G. Costa, C. Stirling, Weak and strong fairness in CCS, Information and Computation 73, 1987, pp. 207-244.

[D] E.W. Duskstra, A discipline of programming, Prentice-Hall, 1976.

[DM] P. DEgano, U. MONTANARI, Liveness properties as convergence in metric spaces, Proceedings of the sixteenth annual ACM Symposium on Theory of Computing, Washington, D.C., 1984, pp. 31-38.

[E] R. Engelking, General Tolology, Allyn and Bacon, 1966.

[F] N. Francez, Faimess, Springer-Verlag, 1986.

[LPS] D. Lehmann, A. Pnueli, J. Stavi, Impartiality, justness and fairness: the ethics of concurrent termination, Proceedings 8th ICALP, Acre, July 1981 (O. Kariv, S. Even, Eds.), Lecture Notes in Computer Science 115, Springer-Verlag, 1981.

[N] M. NIVAT, Infinite words, infinite trees, infinite computations, in: Foundations of Computer Science III.2 (J.W. de Bakker, J. van Leeuwen, eds.), Mathematical Centre Tracts 109, Amsterdam, 1979, pp. 3-52. 
[OA] E.-R. OlderoG, K.R. APT, Transformations realizing fairness assumptions for parallel programs, Proceedings STACS 1984, Lecture Notes in Computer Science 166, Springer-Verlag, 1984.

[P] G.D. PlotKIn, A powerdomain for countable nondeterminism, in: Automata, Languages and Programming, Proceedings 9th ICALP, Aarhus, July 1982 (M. Nielsen, E.M. Schmidt, Eds.), Lecture Notes in Computer Science 140, Springer-Verlag, 1982, pp. 418-428. 